TRANSACTIONS OF THE

AMERICAN MATHEMATICAL SOCIETY

Volume 359, Number 12, December 2007, Pages 5725-5759

S 0002-9947(07)04260-2

Article electronically published on July 3, 2007

\title{
THE DISTANCE FUNCTION FROM THE BOUNDARY IN A MINKOWSKI SPACE
}

\author{
GRAZIANO CRASTA AND ANNALISA MALUSA
}

\begin{abstract}
Let the space $\mathbb{R}^{n}$ be endowed with a Minkowski structure $M$ (that is, $M: \mathbb{R}^{n} \rightarrow[0,+\infty)$ is the gauge function of a compact convex set having the origin as an interior point, and with boundary of class $\left.C^{2}\right)$, and let $d^{M}(x, y)$ be the (asymmetric) distance associated to $M$. Given an open domain $\Omega \subset \mathbb{R}^{n}$ of class $C^{2}$, let $d_{\Omega}(x):=\inf \left\{d^{M}(x, y) ; y \in \partial \Omega\right\}$ be the Minkowski distance of a point $x \in \Omega$ from the boundary of $\Omega$. We prove that a suitable extension of $d_{\Omega}$ to $\mathbb{R}^{n}$ (which plays the rôle of a signed Minkowski distance to $\partial \Omega$ ) is of class $C^{2}$ in a tubular neighborhood of $\partial \Omega$, and that $d_{\Omega}$ is of class $C^{2}$ outside the cut locus of $\partial \Omega$ (that is, the closure of the set of points of nondifferentiability of $d_{\Omega}$ in $\Omega$ ). In addition, we prove that the cut locus of $\partial \Omega$ has Lebesgue measure zero, and that $\Omega$ can be decomposed, up to this set of vanishing measure, into geodesics starting from $\partial \Omega$ and going into $\Omega$ along the normal direction (with respect to the Minkowski distance). We compute explicitly the Jacobian determinant of the change of variables that associates to every point $x \in \Omega$ outside the cut locus the pair $\left(p(x), d_{\Omega}(x)\right)$, where $p(x)$ denotes the (unique) projection of $x$ on $\partial \Omega$, and we apply these techniques to the analysis of PDEs of Monge-Kantorovich type arising from problems in optimal transportation theory and shape optimization.
\end{abstract}

\section{INTRODUCTION}

In recent years the study of the distance function from the boundary has attracted the attention of many researchers coming from different areas of mathematical analysis. We mention, among other papers, 15] in the framework of general first-order Hamilton-Jacobi equations and Finsler geometry, 14 and 17 in the case of Riemannian manifolds, 10] and 21 for the point of view of nonsmooth analysis in Hilbert spaces, 13. for results applied to the theory of Sobolev spaces, and 9 for applications to causality theory.

The results of the present paper are mainly motivated by their applications to the analysis of PDEs. Let $\Omega \subset \mathbb{R}^{n}, n \geq 2$, be a smooth open domain (i.e., a nonempty open bounded connected subset of $\mathbb{R}^{n}$, with sufficiently smooth boundary), and let $H: \bar{\Omega} \times \mathbb{R}^{n} \rightarrow \mathbb{R}$ be a smooth hamiltonian, such that for every $x \in \bar{\Omega}$ the sublevel $K(x):=\left\{p \in \mathbb{R}^{n} ; H(x, p) \leq 1\right\}$ is a compact convex set having the origin as an interior point, with $C^{2}$ boundary and with strictly positive principal curvatures at

Received by the editors May 31, 2005.

2000 Mathematics Subject Classification. Primary 35A30; Secondary 26B05, 32F45, 35C05, 49L25, 58J60.

Key words and phrases. Distance function, Minkowski structure, cut locus, Hamilton-Jacobi equations.

(C)2007 American Mathematical Society 
any point $\left(K(x) \in C_{+}^{2}\right.$ for short). It is well known that the function

$$
F(x, v):=\max \{\langle v, p\rangle ; p \in K(x)\}, \quad x \in \bar{\Omega}, v \in \mathbb{R}^{n}
$$

is a Finsler structure on $\bar{\Omega}$ (see [3]), that is, a smooth function defined in $\bar{\Omega} \times\left(\mathbb{R}^{n} \backslash\{0\}\right)$, positively 1 -homogeneous with respect to $v$, and such that the Hessian matrix $\partial_{v_{i} v_{j}}^{2} F^{2}$ is positive definite at every point of $\bar{\Omega} \times\left(\mathbb{R}^{n} \backslash\{0\}\right)$.

For every pair of points $x, y \in \bar{\Omega}$, we can define the geodesic distance from $y$ to $x$ as

$$
L(x, y):=\inf \left\{\int_{0}^{1} F(\xi(t), \dot{\xi}(t)) d t ; \xi \in S_{x, y}\right\},
$$

where $S_{x, y}$ is the set of all Lipschitz curves $\xi:[0,1] \rightarrow \bar{\Omega}$ such that $\xi(0)=y$ and $\xi(1)=x$. It is well known that the distance from the boundary of $\Omega$, defined by

$$
d_{\Omega}(x):=\inf _{y \in \partial \Omega} L(x, y), \quad x \in \bar{\Omega}
$$

is the unique viscosity solution of the Hamilton-Jacobi equation

$$
\begin{cases}H(x, D u(x))=1 & \text { in } \Omega, \\ u=0 & \text { on } \partial \Omega\end{cases}
$$

(see 16]). Moreover, for every $x \in \Omega$ the infimum in (1.1) is achieved at a point $y \in \partial \Omega$ that can be joined to $x$ by a geodesic going into $\Omega$ and starting from $y$ along the "normal" direction to $\partial \Omega$ (see [15]).

It is worth remarking that, in the simple example $H(x, p)=|p|$, then $F(x, v)=$ $|v|,\left(\mathbb{R}^{n}, F\right)$ is the standard Euclidean space, and (1.2) is the eikonal equation. Hence $d_{\Omega}$ is the Euclidean distance of $x$ from $\partial \Omega$, that is,

$$
d_{\Omega}(x)=\inf _{y \in \partial \Omega}|x-y|, \quad x \in \bar{\Omega} .
$$

Another case, that will be the one considered in our analysis, concerns autonomous Hamiltonians of the form $H(x, p)=\rho(p)$, where $\rho(p):=\inf \{t \geq 0 ; p \in$ $t K\}$ is the gauge function of a fixed compact convex set $K \in C_{+}^{2}$. In this case the function $F(x, v)$ coincides with the gauge function $\rho^{0}(v)$ of the polar set $K^{0}$ of $K$, and $\left(\mathbb{R}^{n}, F\right)$ is a Minkowski space (see [3, Chap. 14]). The function

$$
d_{\Omega}(x)=\inf _{y \in \partial \Omega} \rho^{0}(x-y), \quad x \in \bar{\Omega},
$$

which is the unique viscosity solution of

$$
\begin{cases}\rho(D u)=1 & \text { in } \Omega, \\ u=0 & \text { on } \partial \Omega,\end{cases}
$$

will be called the Minkowski distance from $\partial \Omega$. This nonsymmetric distance function from the boundary is exactly the object we shall deal with in this paper.

Our results can be divided into two main groups, the first one devoted to the differentiability properties of $d_{\Omega}$, and the other one on the regularity of the closure $\bar{\Sigma}$ of the set $\Sigma$ of those points in $\Omega$ where $d_{\Omega}$ is not differentiable.

Concerning the differentiability of $d_{\Omega}$, we consider a signed distance $d_{\Omega}^{s}$, defined in the whole $\mathbb{R}^{n}$, which extends $d_{\Omega}$, and we prove that $d_{\Omega}^{s}$ is of class $C^{2}$ in a tubular neighborhood of $\partial \Omega$ (see Theorem 4.16). This result provides a good definition of $D d_{\Omega}$ and $D^{2} d_{\Omega}$ on $\partial \Omega$. For every $y \in \partial \Omega, D d_{\Omega}(y)$ is proportional to the inward normal $\nu(y)$ to $\partial \Omega$ at $y$ (see Lemma 4.3). Moreover, due to the Minkowskian 
structure of the space, for every $x \in \Omega$ and for every projection $x_{0}$ of $x$ on $\partial \Omega$ the geodesic jointing $x_{0}$ with $x$ is a segment starting from $x_{0}$ and going into $\Omega$ along the "normal" direction, which is $D \rho\left(\nu\left(x_{0}\right)\right.$ ) (see Proposition 4.4). Thanks to these regularity results, for every $y \in \partial \Omega$ we are able to define and study a linear mapping acting on the tangent space to $\partial \Omega$ at $y$ which plays the rôle of the Weingarten map in Riemannian geometry; that is, it has real eigenvalues which are normal curvatures with respect to the Minkowski distance.

For what concerns the singular set, a well known regularity result is the rectifiability of $\Sigma$. Namely, since $d_{\Omega}$ is a locally semiconcave function on $\Omega$ (see [4, 8]), then $\Sigma$ is $C^{2}$-rectifiable (see [1]); that is, it can be covered by a countable family of embedded $C^{2}$ manifolds of dimension $n-1$, with the exception of a set of vanishing $\mathcal{H}^{n-1}$ measure (here $\mathcal{H}^{s}$ denotes the $s$-dimensional Hausdorff measure). In general, this is the best regularity result that we can expect for $\Sigma$, and it remains valid even without any smoothness assumption on $\partial \Omega$.

On the other hand, the set $\bar{\Sigma}$ may behave badly. In [17] a domain $\Omega \subset \mathbb{R}^{2}$, of class $C^{1,1}$, is exhibited such that $\bar{\Sigma}$ has positive Lebesgue measure. (In the same paper the authors prove a rectifiability result in a Riemannian setting.) $\mathrm{Li}$ and Nirenberg in 15 have shown that, if $\Omega$ is of class $C^{2,1}$ and $F$ is of class $C^{\infty}$, then $\mathcal{H}^{n-1}(\bar{\Sigma})$ is finite; hence its Hausdorff dimension does not exceed $n-1$. For related results in Finsler spaces, see also [8, $\S 6]$ and [18.

Our result in this direction is the following (see Theorem 6.11): If $\Omega$ and $F(x, p)=\rho^{0}(p)$ are of class $C^{2, \alpha}$, for some $\alpha \in[0,1]$, then the Hausdorff dimension of $\bar{\Sigma}$ does not exceed $n-\alpha$. In the case $\alpha=0$ (that is, $\Omega$ and $\rho^{0}$ of class $C^{2}$ ), we show that $\bar{\Sigma}$ has vanishing Lebesgue measure and that $d_{\Omega}$ is of class $C^{2}$ on $\Omega \backslash \bar{\Sigma}$ (see Corollary 6.9 and Theorem6.10). Finally, the set $\Omega \backslash \bar{\Sigma}$ can be decomposed into geodesics (segments) starting from $\partial \Omega$ and going into $\Omega$ along the normal direction. We compute explicitly the Jacobian determinant of the change of variables which associates to every point $x \in \Omega \backslash \bar{\Sigma}$ the pair $\left(p(x), d_{\Omega}(x)\right)$, where $p(x)$ denotes the (unique) projection of $x$ on $\partial \Omega$, and we are able to perform a change of variables in multiple integrals in $\Omega$ (see Theorem 7.1).

As an application of these results, we consider the following system of PDEs of Monge-Kantorovich type:

$$
\begin{cases}-\operatorname{div}(v D \rho(D u))=f & \text { in } \Omega, \\ \rho(D u) \leq 1 & \text { in } \Omega, \\ \rho(D u)=1 & \text { in }\{v>0\},\end{cases}
$$

where the source $f \geq 0$ is a continuous function in $\Omega$, complemented with the conditions

$$
\begin{cases}u \geq 0, v \geq 0 & \text { in } \Omega, \\ u=0 & \text { on } \partial \Omega .\end{cases}
$$

The first equation in (1.3) has to be understood in the sense of distributions, whereas $u$ is a viscosity solution to the Hamilton-Jacobi equation $\rho(D u)=1$ in the set $\{v>0\}$. We look for a solution $(u, v)$ to (1.3)-(1.4) in the class of continuous and nonnegative functions.

This system of PDEs arises in problems of shape optimization (see [5]). In the case $\rho(\xi)=|\xi|$, (1.3)-(1.4) describes the stationary solutions of models in granular matter theory (see [6]). 
In section 7, using a change of variables formula, we shall explicitly construct a nonnegative and continuous function $v_{f}$ (defined in (7.7)) such that the pair $\left(d_{\Omega}, v_{f}\right)$ is a solution to (1.3)-(1.4), extending a result proved in [6] in the case $\rho(\xi)=|\xi|$.

\section{Notation AND PRELIMINARIES}

2.1. Basic notation. The standard scalar product of two vectors $x, y \in \mathbb{R}^{n}$ is denoted by $\langle x, y\rangle$, and $|x|$ denotes the Euclidean norm of $x \in \mathbb{R}^{n}$. By $S^{n-1}$ we denote the set of unit vectors of $\mathbb{R}^{n}$, and by $\mathcal{M}_{k}$ the set of $k \times k$ square matrices. We shall denote by $\left(e_{1}, \ldots, e_{n}\right)$ the standard basis of $\mathbb{R}^{n}$. Given two points $x, y \in \mathbb{R}^{n}$, $[x, y]$ will denote the closed segment joining $x$ to $y$, while $(x, y)$ will denote the same segment without the endpoints. If $A, B \subset \mathbb{R}^{n}, x \in \mathbb{R}^{n}$ and $t \in \mathbb{R}$, we define $A+x=\{a+x ; a \in A\}, t A=\{t a ; a \in A\}$ and $A+B=\{a+b ; a \in A, b \in B\}$.

As is customary, $B_{r}\left(x_{0}\right)$ and $\bar{B}_{r}\left(x_{0}\right)$ are respectively the open and the closed balls centered at $x_{0}$ and with radius $r>0$. Given two vectors $v, w \in \mathbb{R}^{n}$, the symbol $v \otimes w$ will denote their tensor product, that is, the linear application from $\mathbb{R}^{n}$ to $\mathbb{R}^{n}$ defined by $(v \otimes w)(x)=v\langle w, x\rangle$.

We shall denote by $\mathcal{L}^{n}(A)$ and $\mathcal{H}^{s}(A)$, respectively, the Lebesgue measure and the $s$-dimensional Hausdorff measure of a set $A \subset \mathbb{R}^{n}$.

Given $A \subset \mathbb{R}^{n}$, we shall denote by $\operatorname{Lip}(A), C(A)$ and $C^{k}(A), k \in \mathbb{N}$, the sets of functions $u: A \rightarrow \mathbb{R}$ that are respectively Lipschitz continuous, continuous and $k$-times continuously differentiable in $A$. Moreover, $C^{\infty}(A)$ will denote the set of functions of class $C^{k}(A)$ for every $k \in \mathbb{N}$, while $C^{k, \alpha}(A)$ will be the set of functions of class $C^{k}(A)$ with Hölder continuous $k$-th partial derivatives with exponent $\alpha \in$ $[0,1]$.

A bounded open set $A \subset \mathbb{R}^{n}$ (or, equivalently, its closure $\bar{A}$ or its boundary $\partial A$ ) is of class $C^{k}, k \in \mathbb{N}$, if for every point $x_{0} \in \partial A$ there exists a ball $B=B_{r}\left(x_{0}\right)$ and a one-to-one mapping $\psi: B \rightarrow D$ such that $\psi \in C^{k}(B), \psi^{-1} \in C^{k}(D), \psi(B \cap A) \subseteq$ $\left\{x \in \mathbb{R}^{n} ; x_{n}>0\right\}, \psi(B \cap \partial A) \subseteq\left\{x \in \mathbb{R}^{n} ; x_{n}=0\right\}$. If the maps $\psi$ and $\psi^{-1}$ are of class $C^{\infty}$ or $C^{k, \alpha}(k \in \mathbb{N}, \alpha \in[0,1])$, then $A$ is said to be of class $C^{\infty}$ or $C^{k, \alpha}$ respectively.

2.2. Differential geometry. We recall briefly some elementary facts from the differential geometry of hypersurfaces of class $C^{2}$ (see e.g. 25). Let $A \subset \mathbb{R}^{n}$ be a bounded open set of class $C^{2}$. For every $x \in \partial A$, we denote respectively by $\nu(x)$ and $T_{x} A$ the unique inward unit normal vector and the tangent space of $\partial A$ at $x$. The map $\nu: \partial A \rightarrow S^{n-1}$ is called the spherical image map (or Gauss map). It is of class $C^{1}$ and, for every $x \in \partial A$, its differential $d \nu_{x}$ maps the tangent space $T_{x} A$ into itself. The linear map $L_{x}:=-d \nu_{x}: T_{x} A \rightarrow T_{x} A$ is called the Weingarten map. The bilinear form defined on $T_{x} A$ by $S_{x}(v, w)=\left\langle L_{x} v, w\right\rangle, v, w \in T_{x} A$, is the second fundamental form of $\partial A$ at $x$. The geometric meaning of the Weingarten map is the following. For every $v \in T_{x} A$ with unit norm, $S_{x}(v, v)$ is equal to the normal curvature of $\partial A$ at $x$ in the direction $v$, that is, $S_{x}(v, v)=\langle\ddot{\xi}(0), \nu(x)\rangle$, where $\xi(t)$ is any parameterized curve in $\partial A$ such that $\xi(0)=x$ and $\dot{\xi}(0)=v$. The eigenvalues $\kappa_{1}(x), \ldots, \kappa_{n-1}(x)$ of the Weingarten map $L_{x}$ are, by definition, the principal curvatures of $\partial A$ at $x$. The corresponding eigenvectors are called the principal directions of $\partial A$ at $x$. It is readily shown that every $\kappa_{i}(x)$ is the normal curvature of $\partial A$ at $x$ in the direction of the corresponding eigenvector. From the $C^{2}$ 
regularity assumption on the manifold $\partial A$, it follows that the principal curvatures of $\partial A$ are continuous functions on $\partial A$.

2.3. Convex geometry. By $\mathcal{K}_{0}^{n}$ we denote the class of nonempty, compact, convex subsets of $\mathbb{R}^{n}$ with the origin as an interior point. We shall briefly refer to the elements of $\mathcal{K}_{0}^{n}$ as convex bodies. The polar body of a convex body $K \in \mathcal{K}_{0}^{n}$ is defined by

$$
K^{0}=\left\{p \in \mathbb{R}^{n} ;\langle p, x\rangle \leq 1 \forall x \in K\right\} .
$$

We recall that, if $K \in \mathcal{K}_{0}^{n}$, then $K^{0} \in \mathcal{K}_{0}^{n}$ and $K^{00}=\left(K^{0}\right)^{0}=K$ (see [23, Thm. 1.6.1]).

Given $K \in \mathcal{K}_{0}^{n}$ we define its gauge function as

$$
\rho_{K}(\xi)=\inf \{t \geq 0 ; \xi \in t K\} .
$$

It is easily seen that

$$
\rho_{K^{0}}(\xi)=\sup \{\langle\xi, p\rangle ; p \in K\} ;
$$

that is, the gauge function of the polar set $K^{0}$ coincides with the support function of the set $K$. Let $0<c_{1} \leq c_{2}$ be such that $\bar{B}_{c_{2}^{-1}}(0) \subseteq K \subseteq \bar{B}_{c_{1}^{-1}}(0)$. Upon observing that $\xi / \rho_{K}(\xi) \in K$ for every $\xi \neq 0$, we get

$$
c_{1}|\xi| \leq \rho_{K}(\xi) \leq c_{2}|\xi|, \quad \forall \xi \in \mathbb{R}^{n} .
$$

We say that $K \in \mathcal{K}_{0}^{n}$ is of class $C_{+}^{2}$ if $\partial K$ is of class $C^{2}$ and all the principal curvatures are strictly positive functions on $\partial K$. In this case, we define the $i$-th principal radius of curvature at $x \in \partial K$ as the reciprocal of the $i$-th principal curvature of $\partial K$ at $x$. We remark that, if $K$ is of class $C_{+}^{2}$, then $K^{0}$ is also of class $C_{+}^{2}$ (see [23, p. 111]). Moreover, a convex body of class $C_{+}^{2}$ is necessarily a strictly convex set.

Throughout the paper we assume that

$$
K \in \mathcal{K}_{0}^{n} \text { is of class } C_{+}^{2} .
$$

Since $K$ will be kept fixed, from now on we shall use the notation $\rho=\rho_{K}$ and $\rho^{0}=\rho_{K^{0}}$.

We collect here some known properties of $\rho$ and $\rho^{0}$ that will be frequently used in the sequel.

Theorem 2.1. Let $K$ satisfy (2.2). Then the following hold:

(i) The functions $\rho$ and $\rho^{0}$ are convex, positively 1 -homogeneous in $\mathbb{R}^{n}$, and of class $C^{2}$ in $\mathbb{R}^{n} \backslash\{0\}$. As a consequence,

$$
\begin{gathered}
\rho(t \xi)=t \rho(\xi), \quad D \rho(t \xi)=D \rho(\xi), \quad D^{2} \rho(t \xi)=\frac{1}{t} D^{2} \rho(\xi), \\
\rho^{0}(t \xi)=t \rho^{0}(\xi), D \rho^{0}(t \xi)=D \rho^{0}(\xi), \quad D^{2} \rho^{0}(t \xi)=\frac{1}{t} D^{2} \rho^{0}(\xi),
\end{gathered}
$$

for every $\xi \in \mathbb{R}^{n} \backslash\{0\}$ and $t>0$. Moreover

$$
\begin{gathered}
\langle D \rho(\xi), \xi\rangle=\rho(\xi), \quad\left\langle D \rho^{0}(\xi), \xi\right\rangle=\rho^{0}(\xi), \quad \forall \xi \in \mathbb{R}^{n} \backslash\{0\}, \\
D^{2} \rho(\xi) \xi=0, \quad D^{2} \rho^{0}(\xi) \xi=0, \quad \forall \xi \in \mathbb{R}^{n} \backslash\{0\} .
\end{gathered}
$$

(ii) For every $\xi, \eta \in \mathbb{R}^{n}$, we have

$$
\rho(\xi+\eta) \leq \rho(\xi)+\rho(\eta), \quad \rho^{0}(\xi+\eta) \leq \rho^{0}(\xi)+\rho^{0}(\eta),
$$

and equality holds if and only if $\xi$ and $\eta$ belong to the same ray, that is, $\xi=\lambda \eta$ or $\eta=\lambda \xi$ for some $\lambda \geq 0$. 
(iii) For every $\xi \neq 0, D \rho(\xi)$ belongs to $\partial K^{0}$, while $D \rho^{0}(\xi)$ belongs to $\partial K$. More precisely, $D \rho(\xi)$ is the unique point of $\partial K^{0}$ such that

$$
\langle D \rho(\xi), \xi\rangle=\rho(\xi) \text {, and }\langle x, \xi\rangle<\rho(\xi) \forall x \in K^{0}, x \neq D \rho(\xi) \text {. }
$$

Symmetrically, the gradient of $D \rho^{0}(\xi)$ is the unique point of $\partial K$ such that

$$
\left\langle D \rho^{0}(\xi), \xi\right\rangle=\rho^{0}(\xi) \text {, and }\langle x, \xi\rangle<\rho^{0}(\xi) \forall x \in K, x \neq D \rho^{0}(\xi) \text {. }
$$

(iv) The eigenvalues of the second differential $D^{2} \rho$ at $\nu \in S^{n-1}$ are 0 , with corresponding eigenvector $\nu$, and the principal radii of curvature of $\partial K^{0}$ at the unique point $p \in \partial K^{0}$ at which $\nu$ is attained as an outward normal vector. Symmetrically, the eigenvalues of $D^{2} \rho^{0}$ at $\nu \in S^{n-1}$ are 0 , with corresponding eigenvector $\nu$, and the principal radii of curvature of $\partial K$ at the unique point $p \in \partial K$ at which $\nu$ is attained as an outward normal vector.

Proof. (i) The convexity and the positive 1-homogeneity are a consequence of the definition. The $C^{2}$ regularity in $\mathbb{R}^{n} \backslash\{0\}$ is proved in [23, p. 106]. The identities follow upon observing that $D \rho$ and $D \rho^{0}$ are positively 0-homogeneous, whereas $D^{2} \rho$ and $D^{2} \rho^{0}$ are positively (-1)-homogeneous. The identity (2.3) is Euler's formula for positively 1-homogeneous functions, whereas (2.4) can be obtained by differentiating (2.3). $K^{0}$.

(ii) The proof follows from convexity of $\rho$ and $\rho^{0}$ and strict convexity of $K$ and

(iii-iv) See 23, Corollaries 1.7.3 and 2.5.2.

Lemma 2.2. Let $K$ satisfy (2.2). Then the identities

$$
D \rho^{0}(D \rho(\xi))=\frac{\xi}{\rho(\xi)}, \quad D \rho\left(D \rho^{0}(\xi)\right)=\frac{\xi}{\rho^{0}(\xi)}
$$

hold for every $\xi \in \mathbb{R}^{n} \backslash\{0\}$.

Proof. For fixed $\xi \in \mathbb{R}^{n} \backslash\{0\}$, by Theorem 2.1(iii) we have that $D \rho(\xi) \in \partial K^{0}$, $D \rho^{0}(D \rho(\xi)) \in \partial K$; hence $\rho^{0}(D \rho(\xi))=1$ and

$$
\begin{aligned}
& \left\langle D \rho^{0}(D \rho(\xi)), D \rho(\xi)\right\rangle=1, \\
& \langle x, D \rho(\xi)\rangle<1 \quad \forall x \in K, x \neq D \rho^{0}(D \rho(\xi)) .
\end{aligned}
$$

On the other hand by (2.3), the point $\xi / \rho(\xi) \in \partial K$ satisfies

$$
\left\langle D \rho(\xi), \frac{\xi}{\rho(\xi)}\right\rangle=1
$$

which, together with (2.7), implies the first identity in (2.6). The second identity can be obtained from the first by interchanging the rôles of $K^{0}$ and $K$.

Remark 2.3. It can be checked that Lemma 2.2 holds under the weaker assumption that the sets $K, K^{0} \in \mathcal{K}_{0}^{n}$ are both strictly convex. This is equivalent to requiring that $K$ is a strictly convex body of class $C^{1}$. 
2.4. Nonsmooth analysis. Let us recall the notions of semiconcave function and of viscosity solution of Hamilton-Jacobi equations (see for example 4, 8, 16]). A function $u: A \rightarrow \mathbb{R}$ is said to be semiconcave if there exists a constant $C>0$ such that

$$
\lambda u(x)+(1-\lambda) u(y) \leq u(\lambda x+(1-\lambda) y)+C \lambda(1-\lambda)|x-y|^{2}
$$

for every $\lambda \in[0,1]$ and every pair $x, y \in A$ such that the segment $[x, y]$ is contained in $A$. It is easy to check that this amounts to the concavity of the map $x \mapsto$ $u(x)-C|x|^{2}$ on every convex subset of $A$. As a consequence, if $u$ is semiconcave in $A$, then it is locally Lipschitz continuous in $A$.

Let $A \subset \mathbb{R}^{n}$ be an open set, and let $u: A \rightarrow \mathbb{R}$ be a continuous function. The superdifferential $D^{+} u(x)$ and the subdifferential $D^{-} u(x)$ of $u$ at $x \in A$ are defined by

$$
\begin{aligned}
& D^{+} u(x)=\left\{p \in \mathbb{R}^{n} ; \limsup _{y \rightarrow x, y \in A} \frac{u(y)-u(x)-\langle p, y-x\rangle}{|x-y|} \leq 0\right\}, \\
& D^{-} u(x)=\left\{p \in \mathbb{R}^{n} ; \liminf _{y \rightarrow x, y \in A} \frac{u(y)-u(x)-\langle p, y-x\rangle}{|x-y|} \geq 0\right\} .
\end{aligned}
$$

If $u$ is differentiable at $x$, then $D^{+} u(x)=D^{-} u(x)=\{D u(x)\}$ (see [4, Lemma 1.8]).

Let $H: A \times \mathbb{R} \times \mathbb{R}^{n} \rightarrow \mathbb{R}$ be a continuous function. We say that $u \in C(A)$ is a viscosity solution of

$$
H(x, u, D u)=0 \quad \text { in } A,
$$

if, for every $x \in A$, we have that $H(x, u(x), p) \leq 0$ for every $p \in D^{+} u(x)$ and $H(x, u(x), p) \geq 0$ for every $p \in D^{-} u(x)$.

Example 2.4. Given an open bounded set $A \subset \mathbb{R}^{n}$, the Euclidean distance from the boundary of $A$, defined by

$$
u(x)=\inf _{y \in \partial A}|x-y|, \quad x \in A,
$$

is the unique viscosity solution of the eikonal equation $|D u|=1$ in $A$ satisfying the boundary condition $u=0$ on $\partial A$ (see [4, 16]).

Let $u: A \rightarrow \mathbb{R}$ be a locally Lipschitz function. A vector $p \in \mathbb{R}^{n}$ is a reachable gradient of $u$ at $x \in A$ if there exists a sequence $\left(x_{k}\right)_{k}$ in $A \backslash\{x\}$ converging to $x$, such that $u$ is differentiable at $x_{k}$ for every $k$ and $\left(D u\left(x_{k}\right)\right)_{k}$ converges to $p$. The set of all reachable gradients of $u$ at $x$ is denoted by $D^{*} u(x)$.

Since $u$ is locally Lipschitz, it is easily seen that $D^{*} u(x)$ is compact for every $x \in A$. Moreover, since $u$ is differentiable almost everywhere in $A, D^{*} u(x)$ is nonempty for every $x \in A$.

\section{Minkowski Distance From the Boundary}

In the first part of this section we shall define the Minkowski distance from a closed set $S \subset \mathbb{R}^{n}$ and we shall prove some basic properties. Then we shall specialize to the case $S=\partial \Omega$, where $\Omega \subset \mathbb{R}^{n}$ is a nonempty bounded open set, and we shall study some properties of the Minkowski distance from $\partial \Omega$ in $\Omega$.

We recall that $K \in \mathcal{K}_{0}^{n}$ is a fixed convex body of class $C_{+}^{2}$ with polar set $K^{0}$, and that $\rho, \rho^{0}$ are respectively the gauge functions of $K$ and $K^{0}$.

Throughout this section, $S$ will be a nonempty closed subset of $\mathbb{R}^{n}$. 
Definition 3.1 (Minkowski distance from a set). The Minkowski distance from $S$ is the function $\delta_{S}: \mathbb{R}^{n} \rightarrow \mathbb{R}$ defined by

$$
\delta_{S}(x)=\min _{y \in S} \rho^{0}(x-y), \quad x \in \mathbb{R}^{n} .
$$

Some comments are in order. Let us consider the Euclidean distance from $S$, defined by

$$
d_{S}^{E}(x)=\min _{y \in S}|x-y|, \quad x \in \mathbb{R}^{n} .
$$

It is clear that $d_{S}^{E}(x)$ measures the (Euclidean) length of the shortest segment $[y, x]$ joining $x$ to a point $y \in S$. In the definition of $\delta_{S}$, the Euclidean distance $d^{E}(x, y)=|x-y|$ is replaced by the Minkowski distance $d^{M}(x, y)=\rho^{0}(x-y)$. Besides the name, the function $d^{M}$ is not a distance in the usual sense. Namely, it satisfies

$$
\begin{gathered}
d^{M}(x, y) \geq 0, \text { and } d^{M}(x, y)=0 \text { only if } x=y, \\
d^{M}(x, y) \leq d^{M}(x, z)+d^{M}(z, y)
\end{gathered}
$$

for every $x, y, z \in \mathbb{R}^{n}$, but in general it is not symmetric (unless $K$ is a convex body symmetric with respect to the origin). Hence, in the Minkowski distance the "length" of a segment $[y, x]$, defined by $d^{M}(x, y)=\rho^{0}(x-y)$, does not necessarily coincide with the length of the segment $[x, y]$. The space $\left(\mathbb{R}^{n}, d^{M}\right)$ is said to be a Minkowski space. It is well known that geodesics in Minkowskian spaces are straight lines, so that these spaces are geodesically both forward and backward complete (see [3, Chap. 14]).

Remark 3.2. As we have underlined in the introduction, we are interested in applications to nonlinear PDEs, where the convex body $K$ plays the role of a constraint on the gradients of the admissible functions. More precisely, one often deals with the set of Lipschitz continuous functions $u: A \subset \mathbb{R}^{n} \rightarrow \mathbb{R}$ such that $D u(x) \in K$ for almost every $x \in A$, that is, $\rho(D u(x)) \leq 1$ for almost every $x \in A$. We shall see that the Minkowski distance from $S$ satisfies $\rho\left(D \delta_{S}(x)\right)=1$ for almost every $x \in A:=\mathbb{R}^{n} \backslash S$ (see Proposition 3.3(i), and Theorem 2.1(iii)). For this reason we prefer to define $\delta_{S}$ in terms of $\rho^{0}$ (and hence of $K^{0}$ ) instead of using $\rho$.

Since $S$ is nonempty and closed, for every $x \in \mathbb{R}^{n}$ the set of projections of $x$ in $S$, defined by

$$
\Pi_{S}(x)=\left\{y \in S ; \delta_{S}(x)=\rho^{0}(x-y)\right\},
$$

is nonempty and compact.

In the following proposition we gather some basic properties of the Minkowski distance $\delta_{S}$.

Proposition 3.3. The Minkowski distance $\delta_{S}$ from $S$ is a continuous function in $\mathbb{R}^{n}$, locally semiconcave in $\mathbb{R}^{n} \backslash S$ and, for every $x \notin S$, the following hold.

(i) $\delta_{S}$ is differentiable at $x$ if and only if $\Pi_{S}(x)=\{y\}$ is a singleton; in this case $D \delta_{S}(x)=D \rho^{0}(x-y)$ and

$$
D \rho\left(D \delta_{S}(x)\right)=\frac{x-y}{\rho^{0}(x-y)}=\frac{x-y}{\delta_{S}(x)} .
$$

(ii) If $\Pi_{S}(x)$ is not a singleton, then $D^{+} \delta_{S}(x)$ is the convex hull of the set $\left\{D \rho^{0}(x-y) ; y \in \Pi_{S}(x)\right\}$, while $D^{-} \delta_{S}(x)=\emptyset$. 
(iii) For any $y \in \Pi_{S}(x)$, and for any point $z$ in the segment $(x, y)$ we have $\Pi_{S}(z)=\{y\}$. Hence $\delta_{S}$ is differentiable at every point $z \in(x, y)$ and $D \delta_{S}(z)=D \rho^{0}(x-y)$.

(iv) $D^{*} \delta_{S}(x)=\left\{D \rho^{0}(x-y) ; y \in \Pi_{S}(x)\right\}$.

Proof. The proof below is a straightforward adaptation of Proposition 2.14 in [4] and Corollary 3.4.5 in [8, where the case $K=\bar{B}_{1}(0)$ is considered.

The continuity of $\delta_{S}$ follows from the inequalities

$$
-\rho^{0}(z-x) \leq \delta_{S}(x)-\delta_{S}(z) \leq \rho^{0}(x-z), \quad \forall x, z, \in \mathbb{R}^{n} .
$$

The other properties follow from the general theory concerning the marginal functions (see [4, Proposition 2.13], 8, Proposition 3.4.4]). Let $A=\mathbb{R}^{n} \backslash S$ and $F(y, x)=\rho^{0}(x-y)$, so that $\delta_{S}(x)=\min _{y \in S} F(y, x)$ for every $x \in A$. Since $\rho^{0}$ is of class $C^{2}$ in $\mathbb{R}^{n} \backslash\{0\}$, it is clear that $F(y, x), D_{x} F(y, x)=D \rho^{0}(x-y)$ and $D_{x x} F(y, x)=D^{2} \rho^{0}(x-y)$ are continuous functions in $S \times A$. The local semiconcavity of $\delta_{S}$ in $\mathbb{R}^{n} \backslash S$ is a consequence of the same property for marginal functions proved in [8, Proposition 3.4.1].

If we define the sets

$$
\begin{aligned}
& M(x):=\left\{y \in S ; \delta_{S}(x)=F(y, x)\right\}=\Pi_{S}(x), \\
Y(x):= & \left\{D_{x} F(y, x) ; y \in M(x)\right\}=\left\{D \rho^{0}(x-y) ; y \in \Pi_{S}(x)\right\},
\end{aligned}
$$

properties (i) and (ii) are an immediate consequence of [8, Proposition 3.4.4] and Lemma 2.2

In order to prove (iii), let us start by proving that $y \in \Pi_{S}(z)$ for every $z \in(x, y)$. Namely, if not, there exists $y^{\prime} \in S$ such that $\rho^{0}(z-y)>\rho^{0}\left(z-y^{\prime}\right)$. Since $z \in(x, y)$, by Theorem 2.1(ii) we obtain

$$
\rho^{0}(x-y)=\rho^{0}(x-z)+\rho^{0}(z-y)>\rho^{0}(x-z)+\rho^{0}\left(z-y^{\prime}\right) \geq \rho^{0}\left(x-y^{\prime}\right),
$$

which contradicts the fact that $y \in \Pi_{S}(x)$. The same argument shows that $\Pi_{S}(z)=$ $\{y\}$. Namely, if we suppose that there exists $y^{\prime} \in \Pi_{S}(z), y^{\prime} \neq y$, we have that $x-z$ and $z-y^{\prime}$ are not proportional; hence, again from Theorem 2.1(ii), we get

$$
\rho^{0}\left(x-y^{\prime}\right)<\rho^{0}(x-z)+\rho^{0}\left(z-y^{\prime}\right)=\rho^{0}(x-z)+\rho^{0}(z-y)=\rho^{0}(x-y),
$$

which contradicts $y \in \Pi_{S}(x)$. Now the differentiability of $\delta_{S}$ at $z$ and the fact that $D \delta_{S}(z)=D \rho^{0}(x-y)$ follow from (i) and from the 0-homogeneity of $D \rho^{0}$.

Finally, in order to prove (iv), we have to show that $D^{*} \delta_{S}(x)=Y(x)$. The inclusion $D^{*} \delta_{S}(x) \subset Y(x)$ follows from [8, Proposition 3.4.4]. Now let $p \in Y(x)$, and let us prove that $p \in D^{*} d_{\Omega}(x)$. By definition of $Y(x)$, there exists $y \in \Pi_{S}(x)$ such that $p=D \rho^{0}(x-y)$. From (iii) we have that $\delta_{S}$ is differentiable at any point $z \in(x, y)$, and $\delta_{S}(z)=p$; hence $p$ is a reachable gradient at $x$.

The following lemma, which states that the multifunction $x \mapsto \Pi_{S}(x)$ is sequentially upper semicontinuous, is a straightforward consequence of the continuity of $\rho^{0}$ and $\delta_{S}$.

Lemma 3.4. If $\left(x_{k}\right)_{k} \subset \mathbb{R}^{n}$ is a sequence converging to a point $x \in \mathbb{R}^{n}$, if $y_{k} \in$ $\Pi_{S}\left(x_{k}\right)$ for every $k$, and if the sequence $\left(y_{k}\right)_{k}$ converges to $y$, then $y \in \Pi_{S}(x)$. 
Proposition 3.5. Let $x \notin S$. For $y \in \Pi_{S}(x)$ let us define

$$
\tilde{l}_{y}(x)=\delta_{S}(x) \cdot \sup \left\{t>0 ; y \in \Pi_{S}(y+t(x-y))\right\} \in(0,+\infty],
$$

and, if $\tilde{l}_{y}(x)<+\infty$, the point

$$
m_{y}(x)=y+\tilde{l}_{y}(x) \frac{x-y}{\delta_{S}(x)} .
$$

Then $\tilde{l}_{y}(x)$ and $m_{y}(x)$ (when $\tilde{l}_{y}(x)$ is finite) do not depend on the choice of $y \in$ $\Pi_{S}(x)$.

Proof. Observe that, if $z=y+t(x-y), t>0$, and $y \in \Pi_{S}(z)$, then

$$
\delta_{S}(z)=\rho^{0}(z-y)=t \rho^{0}(x-y)=t \delta_{S}(x) .
$$

From the very definition of $\tilde{l}_{y}(x)$ and Proposition 3.3 (iii), we have that $\tilde{l}_{y}(x) \geq$ $\delta_{S}(x)$, for every $y \in \Pi_{S}(x)$. We have two possibilities:

(i) $\tilde{l}_{y}(x)=\delta_{S}(x)$ for every $y \in \Pi_{S}(x)$;

(ii) $\tilde{l}_{y}(x)>\delta_{S}(x)$ for some $y \in \Pi_{S}(x)$.

In case (i) it is plain that $\tilde{l}_{y}(x)$ does not depend on the choice of $y \in \Pi_{S}(x)$. Furthermore, the right-hand side of (3.4) coincides with $x$ for every $y \in \Pi_{S}(x)$; hence $m_{y}(x)=x$ is also independent of the choice of $y \in \Pi_{S}(x)$. Let us consider case (ii). Let $y \in \Pi_{S}(x)$ such that $\tilde{l}_{y}(x)>\delta_{S}(x)$. By definition of $\tilde{l}_{y}(x)$, there exists $t>1$ such that, setting $z=y+t(x-y), y \in \Pi_{S}(z)$. Since $x \in(y, z)$, from Proposition 3.3 (iii) we have that $\Pi_{S}(x)=\{y\}$ is a singleton. This concludes the proof of the proposition.

Thanks to Proposition 3.5, the function $\tilde{l}: \mathbb{R}^{n} \backslash S \rightarrow(0,+\infty]$,

$$
\tilde{l}(x):=\delta_{S}(x) \cdot \sup \left\{t>0 ; y \in \Pi_{S}(y+t(x-y))\right\} \quad\left(y \in \Pi_{S}(x)\right)
$$

is well defined. The same holds true for the function $m:\left\{x \in \mathbb{R}^{n} \backslash S ; \tilde{l}(x)<\right.$ $+\infty\} \rightarrow \Omega$ defined by

$$
m(x):=y+\tilde{l}(x) \frac{x-y}{\delta_{S}(x)} \quad\left(y \in \Pi_{S}(x)\right) .
$$

Remark 3.6. It is straightforward to see that, given $x \notin S$ and $y \in \Pi_{S}(x)$,

$$
\tilde{l}(x)=\sup \left\{\lambda>0 ; y \in \Pi_{S}\left(x_{\lambda}\right)\right\}, \quad x_{\lambda}:=y+\lambda \frac{x-y}{\rho^{0}(x-y)} .
$$

It is easily checked that $x_{\lambda}=x$ for $\lambda=\delta_{S}(x)$; hence, from Proposition 3.3(iii), $\Pi_{S}\left(x_{\lambda}\right)=\{y\}$ for every $\lambda \in\left[0, \delta_{S}(x)\right)$. As a consequence, we recover the inequality $\tilde{l}(x) \geq \delta_{S}(x)$.

From now on we shall restrict our attention to the case $S=\mathbb{R}^{n} \backslash \Omega$, where $\Omega \subset \mathbb{R}^{n}$ will be a fixed nonempty connected bounded open set.

Definition 3.7 (Minkowski distance from the boundary). The Minkowski distance from the boundary of $\Omega$ is defined by

$$
d_{\Omega}(x)=\delta_{\mathbb{R}^{n} \backslash \Omega}(x)=\inf _{y \in \partial \Omega} \rho^{0}(x-y), \quad x \in \bar{\Omega} .
$$


For every $x \in \bar{\Omega}$ we shall denote by $\Pi(x)=\Pi_{\partial \Omega}(x)$ the set of projections of $x$ in $\partial \Omega$. For every $x_{0} \in \partial \Omega$ we define the proximal normal cone to $\partial \Omega$ at $x_{0}$ as the set

$$
N^{P}\left(x_{0}\right):=\left\{\lambda v ; x_{0}+v \in \Omega, x_{0} \in \Pi\left(x_{0}+v\right), \lambda \geq 0\right\}
$$

(see [10]). In general, $N^{P}\left(x_{0}\right)$ is convex, but it may not be closed.

Definition 3.8 (Singular set). We say that $x \in \Omega$ is a regular point of $\Omega$ if $\Pi(x)$ is a singleton. We say that $x \in \Omega$ is a singular point of $\Omega$ if $x \in \Omega$ is not a regular point. We denote by $\Sigma \subseteq \Omega$ the set of all singular points of $\Omega$.

From Proposition 3.3 (i), $\Sigma$ coincides with the set of points in $\Omega$ at which $d_{\Omega}$ is not differentiable.

Since $\Omega$ does not contain half spaces, it is clear that, for every $x \in \Omega$, the quantity $\tilde{l}(x)$ introduced in (3.5) is finite; hence the point $m(x)$ (see (3.6)) is well defined.

Given $x \in \Omega$, we call a ray through $x$ any closed segment $[y, m(x)]$ where $y \in$ $\Pi(x)$. Thus there is a unique ray through $x \in \Omega$ if and only if $\Pi(x)$ is a singleton, that is, if and only if $x \notin \Sigma$. From Proposition 3.5 it is clear that, in any case, all rays through $x \in \Omega$ have the same length $\tilde{l}(x)$ in the Minkowski distance. Observe that the length of a ray $[y, m(x)]$ is measured by $\rho^{0}(m(x)-y)$ and, in general, it is different from the length $\rho^{0}(y-m(x))$ of $[m(x), y]$.

In Lemma 3.9 below we prove that $\tilde{l}$ is an upper semicontinuous function in $\Omega$. Furthermore, in Proposition 3.10 we shall define an upper semicontinuous extension $l$ of $\tilde{l}$ to $\bar{\Omega}$.

Lemma 3.9. The function $\tilde{l}: \Omega \rightarrow \mathbb{R}$ defined in (3.5) is upper semicontinuous in $\Omega$.

Proof. We follow the lines of the proof of Proposition 3.7 in [13, where the same property corresponding to the Euclidean distance is proved. By contradiction, assume that there exists a sequence $\left(x_{k}\right)_{k} \subset \Omega$ converging to a point $x \in \Omega$, such that

$$
\lim _{k} \tilde{l}\left(x_{k}\right)>\tilde{l}(x) .
$$

For every $k \in \mathbb{N}$, choose $y_{k} \in \Pi\left(x_{k}\right)$ and define

$$
\lambda_{k}=\min \left\{\frac{\tilde{l}\left(x_{k}\right)}{d_{\Omega}\left(x_{k}\right)}, 2 \frac{\tilde{l}(x)}{d_{\Omega}(x)}\right\} .
$$

The sequence $\left(y_{k}\right)_{k} \subset \partial \Omega$ is clearly bounded. Moreover, since $\tilde{l}\left(x_{k}\right) \geq d_{\Omega}\left(x_{k}\right)$ for every $k \in \mathbb{N}$ (see Remark [3.6), we have $1 \leq \lambda_{k} \leq 2 \tilde{l}(x) / d_{\Omega}(x)$; hence the sequence $\left(\lambda_{k}\right)_{k}$ is also bounded. We can then extract a subsequence, which we do not relabel, such that $\lim _{k} \lambda_{k}=\lambda \geq 1, \lim _{k} y_{k}=y \in \partial \Omega$. Since, by definition of $\tilde{l}\left(x_{k}\right), \lambda_{k} \leq \tilde{l}\left(x_{k}\right) / d_{\Omega}\left(x_{k}\right)$, we have that $y_{k} \in \Pi\left(y_{k}+\lambda_{k}\left(x_{k}-y_{k}\right)\right)$ for every $k \in \mathbb{N}$. Hence, by Lemma 3.4, we infer that $y \in \Pi(y+\lambda(x-y))$. By the definition of $\tilde{l}(x)$ we conclude that $\lambda \leq \tilde{l}(x) / d_{\Omega}(x)$, so that $\lambda_{k}=\tilde{l}\left(x_{k}\right) / d_{\Omega}\left(x_{k}\right)$ for $k$ large enough. Then

that is,

$$
\lambda=\lim _{k} \frac{\tilde{l}\left(x_{k}\right)}{d_{\Omega}\left(x_{k}\right)},
$$

$$
\lim _{k} \tilde{l}\left(x_{k}\right)=\lambda \lim _{k} d_{\Omega}\left(x_{k}\right) \leq \tilde{l}(x),
$$

in contradiction with (3.10). 
Proposition 3.10. The function $l: \bar{\Omega} \rightarrow \mathbb{R}$ defined by

$$
l(x)= \begin{cases}\tilde{l}(x) & \text { if } x \in \Omega, \\ \sup \left\{d_{\Omega}(z) ; z \in \bar{\Omega} \text { and } x \in \Pi(z)\right\} & \text { if } x \in \partial \Omega,\end{cases}
$$

is upper semicontinuous in $\bar{\Omega}$. Here $\tilde{l}: \Omega \rightarrow(0,+\infty)$ is the function defined in (3.5).

Proof. Recalling Lemma 3.9 we have only to prove that $l$ is upper semicontinuous at every point of $\partial \Omega$. Let $x_{0} \in \partial \Omega$. Assume by contradiction that there exists a sequence $\left(x_{k}\right)_{k} \subset \bar{\Omega}$, converging to $x_{0}$, such that

$$
\lim _{k} l\left(x_{k}\right)>l\left(x_{0}\right) .
$$

Upon passing to a subsequence, it is enough to consider the following two cases:

(a) $x_{k} \in \partial \Omega$ for every $k \in \mathbb{N}$;

(b) $x_{k} \in \Omega$ for every $k \in \mathbb{N}$.

In case (a), by the very definition of $l$, for every $k \in \mathbb{N}$ there exists a point $z_{k}$ such that

$$
z_{k} \in \bar{\Omega}, \quad x_{k} \in \Pi\left(z_{k}\right), \quad d_{\Omega}\left(z_{k}\right)>l\left(x_{k}\right)-1 / k \quad(k \geq 1) .
$$

Since $\left(z_{k}\right)_{k}$ is a bounded sequence, there exists a subsequence, which we do not relabel, and a point $z_{0} \in \bar{\Omega}$ such that $\lim _{k} z_{k}=z_{0}$. From the upper semicontinuity of $\Pi$ we have that $x_{0} \in \Pi\left(z_{0}\right)$; hence by (3.13),

$$
l\left(x_{0}\right) \geq d_{\Omega}\left(z_{0}\right) \geq \underset{k}{\limsup } l\left(x_{k}\right),
$$

in contradiction with (3.12).

Consider now case (b). For every $k \in \mathbb{N}$, let $y_{k} \in \Pi\left(x_{k}\right)$. Up to a subsequence we can assume that $\left(y_{k}\right)_{k}$ is convergent. By the upper semicontinuity of $\Pi,\left(y_{k}\right)_{k}$ converges to $x_{0}$, since $\Pi\left(x_{0}\right)=\left\{x_{0}\right\}$. Let us define

$$
\xi_{k}=\frac{x_{k}-y_{k}}{\rho^{0}\left(x_{k}-y_{k}\right)}, \quad k \in \mathbb{N} .
$$

By the characterization (3.7) of $l$, for every $k$ we can choose $\lambda_{k}>l\left(x_{k}\right)-1 / k$, $\lambda_{k}>0$, such that the point $z_{k}=y_{k}+\lambda_{k} \xi_{k}$ satisfies $y_{k} \in \Pi\left(z_{k}\right)$. Since $\left(\lambda_{k}\right)_{k}$ is bounded and $\xi_{k} \in \partial K^{0}$ for every $k$, we can extract another subsequence (which we do not relabel) such that $\lim _{k} \xi_{k}=\xi \in \partial K^{0}$ and $\lim _{k} \lambda_{k}=\lambda$. Then we have $\lim _{k} z_{k}=x_{0}+\lambda \xi$ and, by the upper semicontinuity of $\Pi, x_{0} \in \Pi\left(x_{0}+\lambda \xi\right)$; hence $l\left(x_{0}\right) \geq d_{\Omega}\left(x_{0}+\lambda \xi\right)$. Collecting all the information we obtain

$$
l\left(x_{0}\right) \geq d_{\Omega}\left(x_{0}+\lambda \xi\right)=\lambda=\lim _{k} \lambda_{k} \geq \limsup _{k} l\left(x_{k}\right),
$$

which contradicts (3.12).

Definition 3.11. The point $m(x)$, defined in (3.6), is called the ridge point of $x \in \Omega$. The set

is called the ridge of $\Omega$.

$$
\mathcal{R}=\left\{x \in \bar{\Omega} ; d_{\Omega}(x)=l(x)\right\}
$$

Observe that, if $x \in \mathcal{R}$, then either $x=m(x)$, or $x \in \partial \Omega$ and $x \notin \Pi(y)$ for every $y \in \Omega$. If $x \in \Omega$ is not a ridge point, then $l(x)>d_{\Omega}(x)$, and, from Proposition 3.3(iii), the set $\Pi(x)$ is a singleton; hence $d_{\Omega}$ is differentiable at $x$. Thus the singular set $\Sigma$ is contained in the ridge $\mathcal{R}$ of $\Omega$. We remark that, in general, the two sets do not coincide, as is shown in the following example. 
Example 3.12. Let $\Omega=\left\{\left(x, y \in \mathbb{R}^{2} ; x^{2} / a^{2}+y^{2} / b^{2}<1\right\}\right.$, where $0<b<a$ and $K=\bar{B}_{1}(0)$. The points $P=\left(\left(a^{2}-b^{2}\right) / a, 0\right)$ and $Q=\left(-\left(a^{2}-b^{2}\right) / a, 0\right)$ are the centers of curvature of $\partial \Omega$ at $(a, 0)$ and $(-a, 0)$ respectively. It can be checked that $\mathcal{R}=[Q, P]$ whereas $\Sigma=(Q, P)$.

\section{Distance from the boundary of a Smooth SeT}

Throughout the rest of the paper, we assume that

$\Omega \subset \mathbb{R}^{n}$ is a nonempty, bounded, open connected set of class $C^{2}$.

For every $x_{0} \in \partial \Omega$ we denote by $\kappa_{1}\left(x_{0}\right), \ldots, \kappa_{n-1}\left(x_{0}\right)$ the principal curvatures of $\partial \Omega$ at $x_{0}$, and by $\nu\left(x_{0}\right)$ the inward normal unit vector to $\partial \Omega$ at $x_{0}$.

Since the boundary of $\Omega$ is regular, we will be able to extend $d_{\Omega}$ outside $\bar{\Omega}$ in such a way that this extension turns out to be of class $C^{2}$ in a tubular neighborhood of $\partial \Omega$ (see Theorem 4.16 below). This fact will allow us to define $D d_{\Omega}$ and $D^{2} d_{\Omega}$ on points of $\partial \Omega$. The extension of $d_{\Omega}$ to $\mathbb{R}^{n}$ can be constructed as follows. Let us define

$$
d_{\Omega}^{-}(x)=\inf _{y \in \partial \Omega} \rho^{0}(y-x), \quad x \in \mathbb{R}^{n} \backslash \Omega,
$$

and the signed distance function from $\partial \Omega$,

$$
d_{\Omega}^{s}(x)= \begin{cases}d_{\Omega}(x), & \text { if } x \in \bar{\Omega}, \\ -d_{\Omega}^{-}(x), & \text { if } x \in \mathbb{R}^{n} \backslash \bar{\Omega} .\end{cases}
$$

For every $x \in \mathbb{R}^{n} \backslash \Omega$ we define the set of projections from outside $\Omega$ by

$$
\Pi^{-}(x)=\left\{y \in \partial \Omega ; d_{\Omega}^{-}(x)=\rho^{0}(y-x)\right\},
$$

and we extend the projection operator $\Pi$ to be

$$
\Pi^{s}(x)= \begin{cases}\Pi(x), & \text { if } x \in \bar{\Omega}, \\ \Pi^{-}(x), & \text { if } x \in \mathbb{R}^{n} \backslash \bar{\Omega} .\end{cases}
$$

We define also the set $\Sigma^{-}$of singular points of $d_{\Omega}^{-}$in $\mathbb{R}^{n} \backslash \bar{\Omega}$ and the set $\Sigma^{s}$ of singular points of $d_{\Omega}^{s}$ in $\mathbb{R}^{n}$. We clearly have the inclusion $\Sigma^{s} \subseteq \Sigma \cup \Sigma^{-} \cup \partial \Omega$.

It is worth observing that, in general, $\Pi^{-}(x) \neq \Pi_{\bar{\Omega}}(x)$ for $x \in \mathbb{R}^{n} \backslash \bar{\Omega}$, since $\rho^{0}$ need not be symmetric.

It is clear from the definition that $d_{\Omega}^{-}$is the Minkowski distance from $\partial \Omega$ induced by the gauge function of $-K^{0}$. We can interpret $d_{\Omega}^{-}(x)$ as the Minkowski distance of a point $x \in \mathbb{R}^{n} \backslash \Omega$ to the boundary of $\Omega$.

As in (3.11), we define a function $l^{-}$on $\partial \Omega$ by

$$
l^{-}\left(x_{0}\right)=\sup \left\{d_{\Omega}^{-}(z) ; z \in \mathbb{R}^{n} \backslash \Omega \text {, and } x_{0} \in \Pi^{-}(z)\right\}, \quad x_{0} \in \partial \Omega .
$$

In the following lemma we show that, under our regularity assumption on $\Omega$, the functions $l$ and $l^{-}$are bounded from below by a positive constant on $\partial \Omega$.

Lemma 4.1. Let $\Omega$ satisfy (4.1). Then there exists a positive constant $\mu$ such that $l\left(x_{0}\right), l^{-}\left(x_{0}\right) \geq \mu$ for every $x_{0} \in \partial \Omega$. As a consequence, $\overline{\mathcal{R}} \subseteq \Omega$.

Proof. We shall only prove the assertion concerning $l$, the other being similar. Since $\Omega$ is of class $C^{2}$, then it satisfies a uniform interior sphere condition of radius $r>0$; that is, for every boundary point $x_{0} \in \partial \Omega$ there exists $z_{0} \in \Omega$ such that $x_{0} \in \bar{B}_{r}\left(z_{0}\right) \subset \bar{\Omega}$. Let $R>0$ denote the maximum of the principal radii of 
curvature of $\partial K^{0}$, so that $K^{0}$ (and $-K^{0}$ ) slides freely inside $\bar{B}_{R}$; that is, for every boundary point $y \in \partial B_{R}$ there exists $z \in B_{R}$ such that $y \in z-K^{0} \subset \bar{B}_{R}$ (see [23. Corollary 3.2.10]). We are going to prove that, for $\mu=r / R,-\mu K^{0}$ slides freely inside $\Omega$. Let $x_{0} \in \partial \Omega$. We have to show that there exists $z \in \Omega$ such that $x_{0} \in z-\mu K^{0} \subset \bar{\Omega}$. From the uniform interior sphere condition, there exists $z_{0} \in \Omega$ such that $x_{0} \in \bar{B}_{r}\left(z_{0}\right) \subset \bar{\Omega}$. On the other hand, $-\mu K^{0}$ slides freely inside $\mu \bar{B}_{R}=\bar{B}_{r}$. Thus, there exists $z \in \bar{B}_{r}\left(z_{0}\right)$ such that $x_{0} \in z-\mu K^{0} \subset \bar{B}_{r}\left(z_{0}\right)$. We have proved that for every $x_{0} \in \partial \Omega$ there exists $z \in \Omega$ such that $x_{0} \in z-\mu K^{0} \subset \bar{\Omega}$. Then $\mu=\rho^{0}\left(z-x_{0}\right) \leq \rho^{0}(z-y)$ for every $y \in \partial \Omega$, that is, $x_{0} \in \Pi(z)$ and $d_{\Omega}(z)=\mu$. Hence $l\left(x_{0}\right) \geq \mu$.

Remark 4.2. As a consequence of Lemma 4.1, we obtain that, for $x_{0} \in \partial \Omega$, the supremum in the definition (3.11) of $l\left(x_{0}\right)$ is achieved by a point $z \in \Omega$. Namely, from the continuity of $d_{\Omega}$ and the upper semicontinuity of $\Pi$, the supremum is achieved at a point $z \in \bar{\Omega}$. Should $z \in \partial \Omega$, then $x_{0} \in \Pi(z)$ would imply $z=x_{0}$ and $l\left(x_{0}\right)=0$, in contradiction with Lemma 4.1 .

In the definition (4.4) of $l^{-}\left(x_{0}\right)$ the supremum need not be achieved, but the argument above shows that we can replace $\mathbb{R}^{n} \backslash \Omega$ by $\mathbb{R}^{n} \backslash \bar{\Omega}$ in the definition of $l^{-}$.

The following result relates the gradient of $d_{\Omega}^{s}$ to the inward normal of $\partial \Omega$.

Lemma 4.3. Let $x \in \mathbb{R}^{n} \backslash \Sigma^{s}, x \notin \partial \Omega$, and let $\Pi^{s}(x)=\left\{x_{0}\right\}$. Then

$$
D d_{\Omega}^{s}(x)=\frac{\nu\left(x_{0}\right)}{\rho\left(\nu\left(x_{0}\right)\right)} .
$$

Proof. Consider first the case $x \in \Omega \backslash \Sigma$. From Lemma 3.5 in [20] we have that $D d_{\Omega}(x)=\lambda \nu\left(x_{0}\right)$ for some $\lambda \geq 0$. On the other hand, from Proposition 3.3(i) we have that $D d_{\Omega}(x)=D \rho^{0}\left(x-x_{0}\right)$, and, from Theorem 2.1(iii), this vector belongs to $\partial K$. Then $1=\rho\left(D d_{\Omega}(x)\right)=\lambda \rho\left(\nu\left(x_{0}\right)\right)$, that is, $\lambda=1 / \rho\left(\nu\left(x_{0}\right)\right)$ and (4.5) follows.

Consider now the case $x \in \mathbb{R}^{n} \backslash\left(\bar{\Omega} \cup \Sigma^{-}\right)$. From Proposition 3.3(i) and Lemma 3.5 in [20] we have that $D \rho_{(-K)^{0}}\left(x-x_{0}\right)=D d_{\Omega}^{-}(x)=-\lambda \nu\left(x_{0}\right)$, for some $\lambda \geq 0$. Upon observing that

$$
(-K)^{0}=-K^{0}, \quad \rho_{-K}(\xi)=\rho_{K}(-\xi), \quad \forall \xi \in \mathbb{R}^{n},
$$

we deduce that $D d_{\Omega}^{s}(x)=D \rho^{0}\left(x_{0}-x\right) \in \partial K$; hence

$$
1=\rho\left(D d_{\Omega}^{s}(x)\right)=\lambda \rho\left(\nu\left(x_{0}\right)\right),
$$

and (4.5) follows.

We are now in a position to characterize the normal directions to the boundary of $\Omega$.

Proposition 4.4. For every $x_{0} \in \partial \Omega$, the proximal normal cone of $\Omega$ at $x_{0}$, defined in (3.9), is given by

$$
N^{P}\left(x_{0}\right)=\left\{\lambda D \rho\left(\nu\left(x_{0}\right)\right) ; \lambda \geq 0\right\},
$$

whereas the proximal normal cone of $\mathbb{R}^{n} \backslash \bar{\Omega}$ at $x_{0}$ with respect to $d_{\Omega}^{-}$is $-N^{P}\left(x_{0}\right)$.

Proof. By Proposition [3.3(i) and Lemma 4.3 the vector $D \rho\left(\nu\left(x_{0}\right)\right)$ belongs to $N^{P}\left(x_{0}\right)$. On the other hand, by the definition of $N^{P}\left(x_{0}\right)$, we have that $w \in$ $N^{P}\left(x_{0}\right) \backslash\{0\}$ if and only if there exists $\mu>0$ such that $x_{0} \in \Pi\left(x_{0}+\mu w\right)$. Then, for 
$\epsilon \in(0, \mu)$, from Proposition 3.3 (iii) we have $\Pi\left(x_{0}+\epsilon w\right)=\left\{x_{0}\right\}$. From Lemma 4.3 and Proposition [3.3(i) we have that

$$
D \rho\left(\nu\left(x_{0}\right)\right)=D \rho\left(D d_{\Omega}\left(x_{0}+\epsilon w\right)\right)=\frac{w}{\rho^{0}(w)} ;
$$

hence $w=\lambda D \rho\left(\nu\left(x_{0}\right)\right)$ with $\lambda=\rho^{0}(w)>0$. The computation of the proximal normal cone of $\mathbb{R}^{n} \backslash \bar{\Omega}$ at $x_{0}$ is similar.

Remark 4.5. As a consequence of Proposition 4.4 and Proposition 3.3(i) it is clear that, from any point $x_{0} \in \partial \Omega, D \rho\left(\nu\left(x_{0}\right)\right)$ is the unique inward "normal" direction with the properties

$$
\Pi^{s}\left[x_{0}+t D \rho\left(\nu\left(x_{0}\right)\right)\right]=\left\{x_{0}\right\}, \quad d_{\Omega}^{s}\left(x_{0}+t D \rho\left(\nu\left(x_{0}\right)\right)\right)=t
$$

for $t$ small enough (see also [15, Lemma 2.2]). In the following proposition we shall show that, under our assumptions, the relations above hold for $t \in\left(-l^{-}\left(x_{0}\right), l\left(x_{0}\right)\right)$. This is a well-known fact in Riemannian geometry (see [22, §III.4]).

Proposition 4.6. For every $x_{0} \in \partial \Omega$, we have that

$$
\Pi^{s}\left(x_{0}+t D \rho\left(\nu\left(x_{0}\right)\right)\right)=\left\{x_{0}\right\} \quad \forall t \in\left(-l^{-}\left(x_{0}\right), l\left(x_{0}\right)\right),
$$

whereas $x_{0} \notin \Pi^{s}\left(x_{0}+t D \rho\left(\nu\left(x_{0}\right)\right)\right)$ for every $t<-l^{-}\left(x_{0}\right)$ and for every $t>l\left(x_{0}\right)$.

Proof. We prove the assertion for $t \geq 0$, the case $t \leq 0$ being similar. For fixed $x_{0} \in \partial \Omega$, by Remark 4.2 there exists $z \in \Omega$ such that $x_{0} \in \Pi(z)$ and $d_{\Omega}\left(x_{0}\right)=l\left(x_{0}\right)$. By Proposition 3.3(iii), $\left\{x_{0}\right\}=\Pi(x)$ for every $x$ in the segment $\left(x_{0}, z\right)$. Moreover, by Proposition 3.3(i) and (4.5), the segment $\left(x_{0}, z\right)$ can be parameterized by

$$
x_{t}=x_{0}+t \frac{z-x_{0}}{\rho^{0}\left(z-x_{0}\right)}=x_{0}+t D \rho\left(\nu\left(x_{0}\right)\right), \quad t \in\left(0, l\left(x_{0}\right)\right),
$$

so that (4.7) holds true. On the other hand, by the very definition of $l\left(x_{0}\right)$, it cannot happen that $x_{0} \in \Pi^{s}\left(x_{t}\right)$ and $t=d_{\Omega}\left(x_{t}\right)>l\left(x_{0}\right)$.

The following result, which will be used in the sequel, states that every point $x \in \Omega \backslash \bar{\Sigma}$ belongs to the interior of the ray $(p(x), m(x))$, where $p(x)$ is the unique projection of $x$ on $\partial \Omega$, and $m(x)$ is the ridge point of $x$ defined in (3.6).

Lemma 4.7. Let $x \in \Omega \backslash \bar{\Sigma}$ and let $p(x)$ denote its unique projection on $\partial \Omega$. Then there exists a point $x_{1} \in \Omega \backslash \bar{\Sigma}$ such that $\Pi\left(x_{1}\right)=\{p(x)\}$ and $x \in\left(p(x), x_{1}\right)$.

Proof. Since $\Omega \backslash \bar{\Sigma}$ is an open set, there exists $r>0$ such that $B_{r}(x) \subset \Omega \backslash \bar{\Sigma}$. For every $z \in B_{r}(x)$ let $p(z)$ denote the unique projection of $z$ on $\partial \Omega$. From Lemma 3.4. the map $p$ is continuous in $B_{r}(x)$. Let $V \subset \partial \Omega$ be a local chart on $\partial \Omega$ containing $p(x)$, and let $\varphi: V \rightarrow \mathbb{R}^{n-1}$ be a local coordinate system, that is, a $C^{2}$ bijection from $V$ to the open set $\mathcal{U}=\varphi(V) \subset \mathbb{R}^{n-1}$. Since $p$ is continuous in $B_{r}(x)$, there exists $\delta \in(0, r)$ such that $p\left(\bar{B}_{\delta}(x)\right) \subset V$. Consider now the map $\psi: S^{n-1} \rightarrow \mathbb{R}^{n-1}$ defined by

$$
\psi(u)=\varphi(p(x+\delta u)), \quad u \in S^{n-1} .
$$

It is clear that this map is continuous, being the composition of continuous maps. From the Borsuk-Ulam theorem (see [11, Corollary 4.2]) there exists $u_{0} \in S^{n-1}$ such that $\psi\left(u_{0}\right)=\psi\left(-u_{0}\right)$. Since $\varphi$ is one-to-one, we deduce that $p\left(x+\delta u_{0}\right)=$ $p\left(x-\delta u_{0}\right)$; that is, the points $x_{1}=x+\delta u_{0}$ and $x_{2}=x-\delta u_{0}$ have the same projection $x_{0} \in \partial \Omega$. From Proposition 3.3(i) and Lemma 4.3 we have that

$$
x_{i}=x_{0}+d_{\Omega}\left(x_{i}\right) D \rho\left(\nu\left(x_{0}\right)\right), \quad i=1,2 .
$$


It is clear that the points $x_{0}, x_{1}$ and $x_{2}$ are collinear. Assume, just to fix the ideas, that $d_{\Omega}\left(x_{1}\right)>d_{\Omega}\left(x_{2}\right)$, that is, $\rho^{0}\left(x_{1}-x_{0}\right)>\rho^{0}\left(x_{2}-x_{0}\right)$, in such a way that $x_{2} \in\left(x_{0}, x_{1}\right)$. Since $x \in\left(x_{1}, x_{2}\right)$ by definition of $x_{1}$ and $x_{2}$, we also have $x \in\left(x_{0}, x_{1}\right)$. Finally, recalling that $\Pi\left(x_{1}\right)=\left\{x_{0}\right\}$, from Proposition 3.3(iii) we conclude that $p(x)=x_{0}$.

Remark 4.8. As a consequence of Lemma 4.7, we have that $l(x)>d_{\Omega}(x)$ for every $x \in \Omega \backslash \bar{\Sigma}$. Hence $\mathcal{R} \subseteq \bar{\Sigma}$.

For many calculations we shall use a preferred system of coordinates in order to parameterize $\partial \Omega$ in a neighborhood of a point $x_{0}$.

Definition 4.9 (Principal coordinate system). Let $x_{0} \in \partial \Omega$. We call principal coordinate system at $x_{0}$ the coordinate system such that $x_{0}=0, e_{n}=\nu\left(x_{0}\right)$ and $e_{i}$ coincides with the $i$-th principal direction of $\partial \Omega$ at $x_{0}, i=1, \ldots, n-1$.

Using the principal coordinate system at $x_{0}, \partial \Omega$ can be parameterized in a neighborhood of $x_{0}$ by a map

$$
X: \mathcal{U} \rightarrow \mathbb{R}^{n}, \quad X(y)=(y, \phi(y)),
$$

where $\mathcal{U} \subset \mathbb{R}^{n-1}$ is a neighborhood of the origin, and $\phi: \mathcal{U} \rightarrow \mathbb{R}$ is a map of class $C^{2}$ satisfying

$$
\phi(0)=0, \quad \frac{\partial \phi}{\partial y_{i}}(0)=0, \quad i=1, \ldots, n-1 .
$$

We shall refer to $X$ as a standard parameterization of $\partial \Omega$ in a neighborhood of $x_{0}$. If we define $N(y)=\nu(X(y)), y \in \mathcal{U}$, then

$$
N(y)=\frac{1}{\sqrt{1+\left|D_{y} \phi(y)\right|^{2}}}\left(-D_{y} \phi(y), 1\right), \quad y \in \mathcal{U},
$$

where $D_{y} \phi=\left(\partial \phi / \partial y_{1}, \ldots, \partial \phi / \partial y_{n-1}\right)$. Since, in the principal coordinate system, the vectors $e_{1}, \ldots, e_{n-1}$ are the principal directions of $\partial \Omega$ at $x_{0}$, we have

$$
N(0)=e_{n}, \quad \frac{\partial N}{\partial y_{i}}(0)=-\kappa_{i} e_{i}, \quad i=1, \ldots, n-1,
$$

where $\kappa_{1}, \ldots, \kappa_{n-1}$ are the principal curvatures of $\partial \Omega$ at $x_{0}$. Starting from the identities

$$
\frac{\partial \phi}{\partial y_{j}}=-N_{j}(y) \sqrt{1+\left|D_{y} \phi(y)\right|^{2}}, \quad j=1, \ldots, n-1,
$$

and differentiating, we easily obtain

$$
\frac{\partial^{2} \phi}{\partial y_{i} \partial y_{j}}(0)=-\frac{\partial N_{j}}{\partial y_{i}}(0)=\kappa_{i} \delta_{i j}, \quad i, j=1, \ldots, n-1,
$$

where $\delta_{i j}$ denotes the Kronecker symbol. Moreover, since

$$
\frac{\partial X}{\partial y_{i}}(y)=e_{i}+\left(0, \frac{\partial \phi}{\partial y_{i}}(y)\right), \quad \frac{\partial^{2} X}{\partial y_{i} \partial y_{j}}(y)=\left(0, \frac{\partial^{2} \phi}{\partial y_{i} \partial y_{j}}(y)\right),
$$

for every $i, j=1, \ldots, n-1$, by (4.8) and (4.10), we get

$$
\frac{\partial X}{\partial y_{i}}(0)=e_{i}, \frac{\partial^{2} X}{\partial y_{i} \partial y_{j}}(0)=\kappa_{i} \delta_{i j} e_{n}, \quad i, j=1, \ldots, n-1 .
$$

In the following lemma we introduce the main tool of our theory, that is, a parameterization of $\Omega$ that will allow us to prove the regularity of the signed distance 
near $\partial \Omega$, as well as the regularity of the set $\bar{\Sigma}$. Moreover, in Section 7 we shall use this parameterization in order to prove a change of variables formula in multiple integrals on $\Omega$.

In the following computations, we extend the notion of inward normal for $x$ in a tubular neighborhood of $\partial \Omega$, by setting $\nu(x)$ to be the gradient of the Euclidean signed distance of $x$ from $\partial \Omega$. In this way $D \nu\left(x_{0}\right)$ is well defined for every $x_{0} \in \partial \Omega$, and $D \nu\left(x_{0}\right) \nu\left(x_{0}\right)=0$.

Lemma 4.10. Let $Y: \mathcal{U} \rightarrow \mathbb{R}^{n}, \mathcal{U} \subset \mathbb{R}^{n-1}$ open, be a local parameterization of $\partial \Omega$ of class $C^{2}$. Let $\Psi: \mathcal{U} \times \mathbb{R} \rightarrow \mathbb{R}^{n}$ be the map defined by

$$
\Psi(y, t)=Y(y)+t D \rho(\nu(Y(y))), \quad(y, t) \in \mathcal{U} \times \mathbb{R} .
$$

Then $\Psi \in C^{1}(\mathcal{U} \times \mathbb{R})$, and

$$
\operatorname{det} D \Psi(y, t)=\rho(\nu(Y(y))) \sqrt{g(y)} \operatorname{det}\left[I+t D^{2} \rho(\nu(Y(y))) D \nu(Y(y))\right]
$$

for every $(y, t) \in \mathcal{U} \times \mathbb{R}$, where

$$
g(y)=\operatorname{det}\left(g_{i j}(y)\right), \quad g_{i j}(y)=\left\langle\frac{\partial Y}{\partial y_{i}}(y), \frac{\partial Y}{\partial y_{j}}(y)\right\rangle, \quad i, j=1, \ldots, n-1,
$$

is the determinant of the matrix of the metric coefficients.

Proof. Since $D \rho \in C^{1}\left(\mathbb{R}^{n} \backslash\{0\}\right)$ and $\nu \circ Y \in C^{1}(\mathcal{U}), \Psi$ is of class $C^{1}$ in $\mathcal{U} \times \mathbb{R}$. Let us fix $y_{0} \in \mathcal{U}$, let $x_{0}=Y\left(y_{0}\right)$, and define

$$
Q=I+t D^{2} \rho\left(\nu\left(x_{0}\right)\right) D \nu\left(x_{0}\right), \quad w_{i}=\frac{\partial Y}{\partial y_{i}}\left(y_{0}\right), \quad i=1, \ldots, n-1 .
$$

We have that

$$
\operatorname{det} D \Psi\left(y_{0}, t\right)=\operatorname{det}\left[Q w_{1}, \ldots, Q w_{n-1}, D \rho\left(\nu\left(x_{0}\right)\right)\right] .
$$

Notice that, for every $i=1, \ldots, n-1,\left\langle w_{i}, \nu\left(x_{0}\right)\right\rangle=0$ and, thanks to (2.4),

$$
\left\langle D^{2} \rho\left(\nu\left(x_{0}\right)\right) D \nu\left(x_{0}\right) w_{i}, \nu\left(x_{0}\right)\right\rangle=\left\langle D \nu\left(x_{0}\right) w_{i}, D^{2} \rho\left(\nu\left(x_{0}\right)\right) \nu\left(x_{0}\right)\right\rangle=0 .
$$

Hence $\left\langle Q w_{i}, \nu\left(x_{0}\right)\right\rangle=0$ for every $i=1, \ldots, n-1$, and $Q \nu\left(x_{0}\right)=\nu\left(x_{0}\right)$. Upon observing that $D \rho\left(\nu\left(x_{0}\right)\right)=w+\rho\left(\nu\left(x_{0}\right)\right) \nu\left(x_{0}\right)$, with $\left\langle w, \nu\left(x_{0}\right)\right\rangle=0$, we get

$$
\begin{aligned}
\operatorname{det} D \Psi\left(y_{0}, t\right) & =\rho\left(\nu\left(x_{0}\right)\right) \operatorname{det}\left[Q w_{1}, \ldots, Q w_{n-1}, \nu\left(x_{0}\right)\right] \\
& =\rho\left(\nu\left(x_{0}\right)\right) \operatorname{det} Q \operatorname{det}\left[w_{1}, \ldots, w_{n-1}, \nu\left(x_{0}\right)\right] .
\end{aligned}
$$

Finally, the matrix $C=\left[w_{1}, \ldots, w_{n-1}, \nu\left(x_{0}\right)\right]$ satisfies

$$
\begin{aligned}
\left(C^{T} C\right)_{i j} & =\left\langle w_{i}, w_{j}\right\rangle, \quad i, j=1, \ldots, n-1, \\
\left(C^{T} C\right)_{k n} & =\left(C^{T} C\right)_{n k}=\delta_{k n}, \quad k=1, \ldots, n .
\end{aligned}
$$

Hence $(\operatorname{det} C)^{2}=\operatorname{det}\left(C^{T} C\right)=g\left(y_{0}\right)$, and (4.13) is proved.

The crucial point in what follows is to have exact information about the degeneracy of the map $\Psi$. The first step in this direction is to simplify the computation of $\operatorname{det}\left[I+t D^{2} \rho(\nu) D \nu\right]$.

Lemma 4.11. For every $x_{0} \in \partial \Omega$, we have that

$$
\operatorname{det}\left[I+t D^{2} \rho\left(\nu\left(x_{0}\right)\right) D \nu\left(x_{0}\right)\right]=\operatorname{det}\left(I_{n-1}-t R D\right),
$$


where $D, R \in \mathcal{M}_{n-1}$ are the symmetric square matrices with entries

$$
D_{i j}=-\kappa_{i}\left(x_{0}\right) \delta_{i j}, \quad R_{i j}=\left\langle D^{2} \rho\left(\nu\left(x_{0}\right)\right) e_{j}, e_{i}\right\rangle, \quad i, j=1, \ldots, n-1,
$$

in the principal coordinate system at $x_{0}$.

Proof. It is enough to observe that, in the principal coordinate system at $x_{0}$, $\left\langle D \nu\left(x_{0}\right) e_{i}, e_{j}\right\rangle=-\kappa_{i} \delta_{i j}$ for every $i, j=1, \ldots, n-1$, whereas, from (2.4),

$$
\left\langle D^{2} \rho\left(\nu\left(x_{0}\right)\right) D \nu\left(x_{0}\right) e_{i}, \nu\left(x_{0}\right)\right\rangle=\left\langle D \nu\left(x_{0}\right) e_{i}, D^{2} \rho\left(\nu\left(x_{0}\right)\right) \nu\left(x_{0}\right)\right\rangle=0
$$

for every $i=1, \ldots, n-1$; hence (4.14) follows.

The equality (4.14) is fruitful, since it allows us to deal with a positive definite matrix $R$ with known inverse matrix, as stated in the following result.

Lemma 4.12. Let $x_{0} \in \partial \Omega$, and let $H \in \mathcal{M}_{n-1}$ be the matrix with entries

$$
H_{i j}=\left\langle D^{2} \rho^{0}\left(D \rho\left(\nu\left(x_{0}\right)\right)\right) e_{j}, e_{i}\right\rangle, \quad i, j=1, \ldots, n-1,
$$

in the principal coordinate system at $x_{0}$. Then

$$
\rho\left(\nu\left(x_{0}\right)\right) R H=I_{n-1},
$$

where $R \in \mathcal{M}_{n-1}$ is the matrix defined in (4.15). As a consequence, $R$ and $H$ are positive definite matrices.

Proof. In order to prove (4.17), let us differentiate the first identity in (2.6) with respect to $\xi$, obtaining

$$
D^{2} \rho^{0}(D \rho(\xi)) D^{2} \rho(\xi)=\frac{1}{\rho(\xi)} I-\frac{1}{\rho(\xi)^{2}} \xi \otimes D \rho(\xi), \quad \forall \xi \neq 0 .
$$

From the symmetry of $D^{2} \rho^{0}$ and $D^{2} \rho$, the adjoint matrix identity can be written as

$$
D^{2} \rho(\xi) D^{2} \rho^{0}(D \rho(\xi))=\frac{1}{\rho(\xi)} I-\frac{1}{\rho(\xi)^{2}} D \rho(\xi) \otimes \xi, \quad \forall \xi \neq 0 .
$$

Notice that, if $v$ is orthogonal to $\nu=\nu\left(x_{0}\right)$, then $(D \rho(\nu) \otimes \nu) v=0$; hence, in the principal coordinate system, for every $i, j=1, \ldots, n-1$,

$$
\begin{aligned}
\frac{1}{\rho(\nu)} \delta_{i j} & =\left\langle D^{2} \rho(\nu) D^{2} \rho^{0}(D \rho(\nu)) e_{i}, e_{j}\right\rangle \\
& =\sum_{l=1}^{n-1}\left\langle D^{2} \rho(\nu) e_{l}, e_{j}\right\rangle\left\langle D^{2} \rho^{0}(D \rho(\nu)) e_{i}, e_{l}\right\rangle,
\end{aligned}
$$

where the last equality is due to the fact that, from (2.4), $D^{2} \rho(\nu) e_{n}=D^{2} \rho(\nu) \nu=0$. The relation above is exactly (4.17).

Recalling that $D^{2} \rho(\nu)$ and $D^{2} \rho^{0}(D \rho(\nu))$ are positive semidefinite (due to the convexity of $\rho$ and $\rho^{0}$ ), it follows that $R$ and $H$ are both positive semidefinite. As a consequence of (4.17), $R$ and $H$ are also invertible; hence they are positive definite.

The fundamental step in the proof of the $C^{2}$ regularity of $d_{\Omega}^{s}$ in a tubular neighborhood of $\partial \Omega$ and of $d_{\Omega}$ in $\bar{\Omega} \backslash \bar{\Sigma}$ is the following.

Theorem 4.13. Let $Y: \mathcal{U} \rightarrow \mathbb{R}^{n}, \mathcal{U} \subset \mathbb{R}^{n-1}$ open, be a local parameterization of $\partial \Omega$ of class $C^{2}$. Let $\Psi: \mathcal{U} \times \mathbb{R} \rightarrow \mathbb{R}^{n}$ be the map defined in (4.12). Then $\operatorname{det} D \Psi(y, t)>0$ for every $y \in \mathcal{U}$ and every $t \in\left(-l^{-}(Y(y)), l(Y(y))\right)$. 
Proof. Let us fix $x_{0} \in \partial \Omega$. There is no loss of generality in assuming $x_{0}=Y(0)$. In the principal coordinate system at $x_{0}$, we have also $x_{0}=0$. We shall denote $\nu=\nu\left(x_{0}\right)=e_{n}$. From Lemmas 4.10 and 4.11, in this coordinate system we have to prove that

$$
\operatorname{det}\left(I_{n-1}-t R D\right)>0, \quad \forall t \in\left(-l^{-}\left(x_{0}\right), l\left(x_{0}\right)\right),
$$

where $D, R \in \mathcal{M}_{n-1}$ are the square matrices defined in (4.15).

Observe that, from (4.17),

$$
I_{n-1}-t R D=\rho(\nu) R H-t R D=\rho(\nu) R\left(H-\frac{t}{\rho(\nu)} D\right),
$$

where $H$ is the matrix defined in (4.16). Since $\operatorname{det}(R)>0$ by Lemma4.12, in order to prove (4.18), it is enough to prove the following claim.

Claim. The matrix $A(t) \in \mathcal{M}_{n-1}$ defined by

$$
A(t)=H-\frac{t}{\rho(\nu)} D, \quad t \in \mathbb{R},
$$

is positive definite for every $t \in\left(-l^{-}\left(x_{0}\right), l\left(x_{0}\right)\right)$.

Proof. For $t=0$ we have $A(0)=H$, which is positive definite by Lemma 4.12, We shall now prove the claim for $t \in\left(0, l\left(x_{0}\right)\right)$, the case $t \in\left(-l\left(x_{0}\right), 0\right)$ being similar.

We start by proving that, for every $t \in\left(0, l\left(x_{0}\right)\right), A(t)$ is positive semidefinite. Let $z_{t}=x_{0}+t D \rho(\nu)$. From Proposition 4.6 we have that $\Pi\left(z_{t}\right)=\left\{x_{0}\right\}$, and $d_{\Omega}\left(z_{t}\right)=t=\rho^{0}\left(z_{t}-x_{0}\right)$. Moreover, the following ball in the Minkowski norm,

$$
B=\left\{x \in \mathbb{R}^{n} ; \rho^{0}\left(z_{t}-x\right)<t\right\}
$$

is contained in $\Omega$, and $x_{0} \in \partial B \cap \partial \Omega$. Thus

$$
h(y):=\rho^{0}\left(z_{t}-X(y)\right) \geq t \quad \forall y \in \mathcal{U}, \quad h(0)=t .
$$

Then, the function $h \in C^{2}(\mathcal{U})$ has a local minimum point at $y=0$; hence its Hessian matrix at $y=0$ must be positive semidefinite. It is straightforward to check that

$$
\begin{aligned}
\frac{\partial h}{\partial y_{i}}(y)= & \left\langle D \rho^{0}\left(z_{t}-X(y)\right), \frac{\partial X}{\partial y_{i}}(y)\right\rangle, \\
\frac{\partial^{2} h}{\partial y_{i} \partial y_{j}}(y)= & \left\langle D^{2} \rho^{0}\left(z_{t}-X(y)\right) \frac{\partial X}{\partial y_{j}}(y), \frac{\partial X}{\partial y_{i}}(y)\right\rangle \\
& +\left\langle D \rho^{0}\left(z_{t}-X(y)\right), \frac{\partial^{2} X}{\partial y_{i} \partial y_{j}}(y)\right\rangle .
\end{aligned}
$$

Using (4.11) we obtain

$$
\frac{\partial^{2} h}{\partial y_{i} \partial y_{j}}(0)=\left\langle D^{2} \rho^{0}\left(z_{t}-x_{0}\right) e_{i}, e_{j}\right\rangle-\kappa_{i} \delta_{i j}\left\langle D \rho^{0}\left(z_{t}-x_{0}\right), \nu\right\rangle .
$$

Since $z_{t}-x_{0}=t D \rho(\nu)$, from the positive 0-homogeneity of $D \rho^{0}$, the positive $(-1)$-homogeneity of $D^{2} \rho^{0}$, and (2.6) we get

$$
D \rho^{0}\left(z_{t}-x_{0}\right)=D \rho^{0}(D \rho(\nu))=\frac{\nu(y)}{\rho(\nu(y))}, \quad D^{2} \rho^{0}\left(z_{t}-x_{0}\right)=\frac{1}{t} D^{2} \rho^{0}(D \rho(\nu)) ;
$$

hence

$$
\frac{\partial^{2} h}{\partial y_{i} \partial y_{j}}(0)=\frac{1}{t}\left\langle D^{2} \rho^{0}(D \rho(\nu)) e_{i}, e_{j}\right\rangle-\frac{\kappa_{i}}{\rho(\nu)} \delta_{i j},
$$


that is, $D^{2} h(0)=(1 / t) H-(1 / \rho(\nu)) D=A(t) / t$. Since the Hessian of $h$ at $y=0$ is a positive semidefinite matrix, we conclude that $A(t)$ is also a positive semidefinite matrix for $t \in\left(0, l\left(x_{0}\right)\right)$.

Let us prove that, in fact, $A(t)$ is positive definite for every $t \in\left(0, l\left(x_{0}\right)\right)$. It is plain that it is equivalent to prove that $\lambda \rho(\nu) H-D$ is positive definite for every $\lambda>1 / l\left(x_{0}\right)$. Let us fix $\lambda>1 / l\left(x_{0}\right)$ and choose $\lambda^{\prime} \in\left(1 / l\left(x_{0}\right), \lambda\right)$, so that $\lambda^{\prime} \rho(\nu) H-D$ is positive semidefinite. Since, by Lemma 4.12, $H$ is positive definite,

$$
\begin{aligned}
\langle(\lambda \rho(\nu) H-D) w, w\rangle & =\left\langle\left(\lambda^{\prime} \rho(\nu) H-D\right) w, w\right\rangle+\left(\lambda-\lambda^{\prime}\right)\langle\rho(\nu) H w, w\rangle \\
& \geq\left(\lambda-\lambda^{\prime}\right)\langle\rho(\nu) H w, w\rangle>0
\end{aligned}
$$

for every $w \neq 0$; that is, $\lambda \rho(\nu) H-D$ is positive definite. This concludes the proof of the claim and of the proposition.

Remark 4.14. It is well known that $d_{\Omega}$ is the unique viscosity solution of the Hamilton-Jacobi equation $\rho\left(D d_{\Omega}\right)=1$ in $\Omega$ vanishing on $\partial \Omega$ (see $4,8,16$ ). The map $\Psi$ defined in (4.12) gives the characteristic curves associated to this PDE. The regularity of the map $\Psi$ proved in Theorem 4.13 will be used in Theorem 4.16 in order to prove the $C^{2}$ regularity of $d_{\Omega}^{s}$ in a tubular neighborhood of $\partial \Omega$. It should be noted that this kind of result in fact follows from the local existence theory for first-order PDEs based on the method of characteristics. Nevertheless the regularity of the map $\Psi$ is an essential tool in order to prove the $C^{2}$ regularity of $d_{\Omega}$ in the whole set $\Omega \backslash \bar{\Sigma}$ (see Theorem 6.10 below).

Corollary 4.15. The ridge set $\mathcal{R}$ has zero Lebesgue measure.

Proof. Let $Y_{k}: \mathcal{U}_{k} \rightarrow \mathbb{R}^{n}, \mathcal{U}_{k} \subset \mathbb{R}^{n-1}$ open, $k=1, \ldots, N$, be local parameterizations of $\partial \Omega$ of class $C^{2}$, such that $\bigcup_{k=1}^{N} Y_{k}\left(\mathcal{U}_{k}\right)=\partial \Omega$. For every $k=1, \ldots, N$, let $\Psi_{k}: \mathcal{U}_{k} \times \mathbb{R} \rightarrow \mathbb{R}^{n}$ be the map

$$
\Psi_{k}(y, t)=Y_{k}(y)+t D \rho\left(\nu\left(Y_{k}(y)\right)\right), \quad(y, t) \in \mathcal{U}_{k} \times \mathbb{R} .
$$

For every $k=1, \ldots, n$, let $U_{k} \subset \mathcal{U}_{k}$ be a compact set such that $\bigcup_{k} Y_{k}\left(U_{k}\right)$ covers $\partial \Omega$, and let

$$
A_{k}=\left\{(y, t) ; y \in U_{k}, t \in\left[0, l\left(Y_{k}(y)\right)\right]\right\} .
$$

From Proposition [3.10, $l$ is an upper semicontinuous function; hence for every $k=1, \ldots, n, A_{k}$ is a compact set and the Lebesgue measure of the graph

$$
\Psi_{k}^{-1}(\mathcal{R}) \cap A_{k}=\left\{(y, t) \in U_{k} \times \mathbb{R} ; t=l\left(Y_{k}(y)\right)\right\}
$$

vanishes. From Theorem 4.13 we know that, for every $k=1, \ldots, N, \Psi_{k} \in$ $C^{1}\left(\mathcal{U}_{k} \times \mathbb{R}\right)$; hence it is Lipschitz continuous on the compact set $A_{k}$. Let $L$ be the maximum of the Lipschitz constants of the functions $\Psi_{1}, \ldots, \Psi_{N}$. Since $\bigcup_{k=1}^{N} \Psi_{k}\left(A_{k}\right)=\bar{\Omega}$, and hence $\mathcal{R} \subseteq \bigcup_{k=1}^{N} \Psi_{k}\left(\Psi_{k}^{-1}(\mathcal{R}) \cap A_{k}\right)$, we finally get

$$
\mathcal{L}^{n}(\mathcal{R}) \leq \sum_{k=1}^{N} \mathcal{L}^{n}\left[\Psi_{k}\left(\Psi_{k}^{-1}(\mathcal{R}) \cap A_{k}\right)\right] \leq L^{n} \sum_{k=1}^{N} \mathcal{L}^{n}\left[\Psi_{k}^{-1}(\mathcal{R}) \cap A_{k}\right]=0
$$

and the proof is complete.

Theorem 4.16 (Regularity of $d_{\Omega}^{s}$ ). The function $d_{\Omega}^{s}$ is of class $C^{2}$ in a tubular neighborhood of $\partial \Omega$ of the form

$$
A_{\mu}=\left\{x \in \mathbb{R}^{n} ;-\mu<d_{\Omega}^{s}(x)<\mu\right\}
$$


for some $\mu>0$. Furthermore, for every $x_{0} \in \partial \Omega$,

$$
D d_{\Omega}^{s}\left(x_{0}+t D \rho\left(\nu\left(x_{0}\right)\right)\right)=D d_{\Omega}^{s}\left(x_{0}\right), \quad t \in(-\mu, \mu),
$$

and then the identity (4.5) holds also for $x \in \partial \Omega$. If $\Omega$ is of class $C^{k, \alpha}$ and $\rho \in C^{k, \alpha}\left(\mathbb{R}^{n} \backslash\{0\}\right)$, for some $k \geq 2$ and $\alpha \in[0,1]$, then $d_{\Omega}^{s}$ is of class $C^{k, \alpha}$ in $A_{\mu}$.

Proof. From Lemma 4.1 there exists $\mu>0$ such that $l\left(x_{0}\right), l^{-}\left(x_{0}\right)>\mu$ for every $x_{0} \in \partial \Omega$; hence, thanks to (4.7), for every point $z \in A_{\mu}$ the projection $\Pi^{s}(z)$ is a singleton. Let $z_{0} \in A_{\mu}$ and prove that $d_{\Omega}^{s}$ is of class $C^{2}$ in a neighborhood of $z_{0}$. Let $\Pi\left(z_{0}\right)=\left\{x_{0}\right\}$, and let $Y: \mathcal{U} \rightarrow \mathbb{R}^{n}, \mathcal{U} \subset \mathbb{R}^{n-1}$ open, be a local parameterization of $\partial \Omega$ in a neighborhood of $x_{0}$ with $Y(0)=x_{0}$. Let $\Psi \in C^{1}(\mathcal{U} \times \mathbb{R})$ be the map defined in (4.12), and let $t_{0}=d_{\Omega}^{s}\left(z_{0}\right) \in(-\mu, \mu)$. From Theorem 4.13 we have that $\operatorname{det} D \Psi\left(0, t_{0}\right)>0$; hence from the inverse mapping theorem it follows that there exists a neighborhood $V \subset A_{\mu}$ of $z_{0}$ such that the maps $y=y(x)$ and $t=t(x)$ are of class $C^{1}(V)$. Since $d_{\Omega}^{s}(x)=t(x)$ for every $x \in V$, this proves that $d_{\Omega}^{s}$ is of class $C^{1}(V)$. Moreover, by (4.5), $D d_{\Omega}^{s}(\Psi(0, t))=\nu\left(x_{0}\right) / \rho\left(\nu\left(x_{0}\right)\right)$ for every $t \in\left(0, t_{0}\right)$; hence by the continuity of $D d_{\Omega}^{s}$ in $V$, and the fact that (4.19) holds in $\left(0, t_{0}\right)$, we have

$$
D d_{\Omega}^{s}\left(x_{0}\right)=\nu\left(x_{0}\right) / \rho\left(\nu\left(x_{0}\right)\right) \quad\left(x_{0} \in \partial \Omega\right) .
$$

Finally, from (4.5) we have that $D d_{\Omega}(x)=N(y(x)) / \rho(N(y(x))), x \in V$, where $N=\nu \circ Y \in C^{1}(\mathcal{U})$; hence $D d_{\Omega} \in C^{1}(V)$, that is, $d_{\Omega} \in C^{2}(V)$.

The last part of the proposition follows from the fact that, if $\Omega$ is of class $C^{k, \alpha}$ and $\rho \in C^{k, \alpha}\left(\mathbb{R}^{n} \backslash\{0\}\right)$, then $\Psi \in C^{k-1, \alpha}(\mathcal{U} \times \mathbb{R})$.

Remark 4.17. As a consequence of Theorem 4.16, we have that $\overline{\Sigma^{s}} \subset \mathbb{R}^{n} \backslash \partial \Omega$. In particular $\bar{\Sigma} \subset \Omega$ and $\overline{\Sigma^{-}} \subset \mathbb{R}^{n} \backslash \bar{\Omega}$.

We conclude this section by giving an explicit representation of the Hessian matrix of $d_{\Omega}$ in $x_{0} \in \partial \Omega$ with respect to the principal coordinate system. From now on, we shall denote $D^{2} d_{\Omega}\left(x_{0}\right)=D^{2} d_{\Omega}^{s}\left(x_{0}\right)$ for every $x_{0} \in \partial \Omega$.

Lemma 4.18. Let $x_{0} \in \partial \Omega$. Then, in the principal coordinate system at $x_{0}$ we have that

$$
\begin{gathered}
\left\langle D^{2} d_{\Omega}\left(x_{0}\right) e_{i}, e_{j}\right\rangle=-\frac{1}{\rho\left(\nu\left(x_{0}\right)\right)} \kappa_{i} \delta_{i j}, \quad i, j=1, \ldots, n-1, \\
\left\langle D^{2} d_{\Omega}\left(x_{0}\right) e_{i}, \nu\left(x_{0}\right)\right\rangle=\frac{\kappa_{i}}{\rho\left(\nu\left(x_{0}\right)\right)^{2}}\left\langle D \rho\left(\nu\left(x_{0}\right)\right), e_{i}\right\rangle, i=1, \ldots, n-1, \\
\left\langle D^{2} d_{\Omega}\left(x_{0}\right) \nu\left(x_{0}\right), \nu\left(x_{0}\right)\right\rangle=-\frac{1}{\rho\left(\nu\left(x_{0}\right)\right)^{3}} \sum_{i=1}^{n-1} \kappa_{i}\left\langle D \rho\left(\nu\left(x_{0}\right)\right), e_{i}\right\rangle^{2} .
\end{gathered}
$$

(Recall that $\nu\left(x_{0}\right)=e_{n}$ in the principal coordinate system.)

Proof. If we differentiate the identity $d_{\Omega}(X(y))=0$ for every $y \in \mathcal{U}$ with respect to $y_{i}, i=1, \ldots, n-1$, we obtain

$$
\left\langle D d_{\Omega}(X(y)), \frac{\partial X}{\partial y_{i}}(y)\right\rangle=0, \quad y \in \mathcal{U} .
$$

A further differentiation with respect to $y_{j}, j=1, \ldots, n-1$, gives

$$
\left\langle D^{2} d_{\Omega}(X(y)) \frac{\partial X}{\partial y_{i}}(y), \frac{\partial X}{\partial y_{j}}(y)\right\rangle+\left\langle D d_{\Omega}(X(y)), \frac{\partial^{2} X}{\partial y_{i} \partial y_{j}}(y)\right\rangle=0, \quad y \in \mathcal{U} .
$$


Evaluating the last expression at $y=0$, and taking into account (4.11) and Lemma 4.3, we obtain (4.21).

In order to prove (4.22) and (4.23), let us differentiate the identity (4.19) with respect to $t$, and evaluate the result at $t=0$. We obtain

$$
D^{2} d_{\Omega}\left(x_{0}\right) D \rho\left(\nu\left(x_{0}\right)\right)=0,
$$

that, upon observing that

$$
D \rho\left(\nu\left(x_{0}\right)\right)=\sum_{l=1}^{n-1}\left\langle D \rho\left(\nu\left(x_{0}\right)\right), e_{l}\right\rangle e_{l}+\rho\left(\nu\left(x_{0}\right)\right) e_{n},
$$

becomes

$$
\sum_{l=1}^{n-1}\left\langle D \rho\left(\nu\left(x_{0}\right)\right), e_{l}\right\rangle D^{2} d_{\Omega}\left(x_{0}\right) e_{l}+\rho\left(\nu\left(x_{0}\right)\right) D^{2} d_{\Omega}\left(x_{0}\right) e_{n}=0 .
$$

Then

$$
\begin{gathered}
\sum_{l=1}^{n-1}\left\langle D \rho\left(\nu\left(x_{0}\right)\right), e_{l}\right\rangle\left\langle D^{2} d_{\Omega}\left(x_{0}\right) e_{l}, e_{i}\right\rangle \\
+\rho\left(\nu\left(x_{0}\right)\right)\left\langle D^{2} d_{\Omega}\left(x_{0}\right) e_{n}, e_{i}\right\rangle=0
\end{gathered}
$$

for every $i=1, \ldots, n$. Hence (4.22) follows from (4.25) and (4.21), while (4.23) follows from (4.25) and (4.22).

Remark 4.19. Formula (4.21) can be written as

$$
\left\langle D^{2} d_{\Omega}\left(x_{0}\right) e_{i}, e_{j}\right\rangle=\frac{1}{\rho\left(\nu\left(x_{0}\right)\right)}\left\langle D e_{i}, e_{j}\right\rangle, \quad i, j=1, \ldots, n-1,
$$

where $D \in \mathcal{M}_{n-1}$ is the matrix defined in (4.15).

\section{5. $\rho$-Curvatures}

Thanks to Theorem 4.16, the function $d_{\Omega}^{s}$ is of class $C^{2}$ on a neighborhood of $\partial \Omega$. We can then define the matrix-valued function

$$
W\left(x_{0}\right)=-D^{2} \rho\left(D d_{\Omega}\left(x_{0}\right)\right) D^{2} d_{\Omega}\left(x_{0}\right), \quad x_{0} \in \partial \Omega,
$$

where $D^{2} d_{\Omega}\left(x_{0}\right) \equiv D^{2} d_{\Omega}^{s}\left(x_{0}\right)$. For any $x_{0} \in \partial \Omega$, let $T_{x_{0}}$ denote the tangent space to $\partial \Omega$ at $x_{0}$. Notice that, if $v \in \mathbb{R}^{n}$, then $W\left(x_{0}\right) v \in T_{x_{0}}$, since from (4.20) and Theorem 2.1(i), (iv)

$$
\begin{aligned}
\left\langle W\left(x_{0}\right) v, \nu\left(x_{0}\right)\right\rangle & =-\left\langle D^{2} d_{\Omega}\left(x_{0}\right) v, D^{2} \rho\left(D d_{\Omega}\left(x_{0}\right)\right) \nu\left(x_{0}\right)\right\rangle \\
& =-\rho\left(\nu\left(x_{0}\right)\right)\left\langle D^{2} d_{\Omega}\left(x_{0}\right) v, D^{2} \rho\left(\nu\left(x_{0}\right)\right) \nu\left(x_{0}\right)\right\rangle=0 .
\end{aligned}
$$

Hence, we can define the map

$$
\bar{W}\left(x_{0}\right): T_{x_{0}} \rightarrow T_{x_{0}}, \quad \bar{W}\left(x_{0}\right) w=W\left(x_{0}\right) w, \quad w \in T_{x_{0}},
$$

that can be identified with a linear application from $\mathbb{R}^{n-1}$ to $\mathbb{R}^{n-1}$. In our setting, the function $\bar{W}$ plays the role of the Weingarten map (see Example 5.6 and Remark 5.9 below). 
Lemma 5.1. Let $x_{0} \in \partial \Omega$. Then, in the principal coordinate system at $x_{0}$, $\bar{W}\left(x_{0}\right)=R D$ where $D$ and $R$ are the symmetric matrices defined in (4.15), one has

$$
\operatorname{det}\left[I-t W\left(x_{0}\right)\right]=\operatorname{det}\left[I_{n-1}-t \bar{W}\left(x_{0}\right)\right], \quad t \in \mathbb{R} .
$$

Furthermore, both determinants are strictly positive for $t \in\left(-l^{-}\left(x_{0}\right), l\left(x_{0}\right)\right)$.

Proof. Consider the principal coordinate system at $x_{0}$. Recall that, from the positive (-1)-homogeneity of $D^{2} \rho$ and (4.20),

$$
D^{2} \rho\left(D d_{\Omega}\left(x_{0}\right)\right)=\rho(\nu) D^{2} \rho(\nu)
$$

where $\nu=\nu\left(x_{0}\right)$. Since $D^{2} \rho(\nu) e_{n}=D^{2} \rho(\nu) \nu=0$ (see (2.4) $)$, we get

$$
\left\langle W\left(x_{0}\right) e_{j}, e_{i}\right\rangle=-\rho(\nu) \sum_{l=1}^{n-1}\left\langle D^{2} \rho(\nu) e_{l}, e_{i}\right\rangle\left\langle D^{2} d_{\Omega}\left(x_{0}\right) e_{j}, e_{l}\right\rangle,
$$

for every $i, j=1, \ldots, n-1$. From Lemma 4.18 we obtain

$$
\left\langle W\left(x_{0}\right) e_{j}, e_{i}\right\rangle=(R D)_{i j}, \quad i, j=1, \ldots, n-1,
$$

where $D$ and $R$ are the symmetric matrices in $\mathcal{M}_{n-1}$ defined in (4.15). Since $T_{x_{0}}$ is spanned by $\left(e_{1}, \ldots, e_{n-1}\right)$, we have that

$$
\bar{W}\left(x_{0}\right)=R D .
$$

Furthermore

$$
\left\langle W\left(x_{0}\right) e_{k}, \nu\right\rangle=-\left\langle D^{2} \rho(\nu) \nu, D^{2} d_{\Omega}\left(x_{0}\right) e_{k}\right\rangle=0, \quad k=1, \ldots, n ;
$$

hence

$$
\operatorname{det}\left[I-t W\left(x_{0}\right)\right]=\operatorname{det}\left[I_{n-1}-t \bar{W}\left(x_{0}\right)\right]=\operatorname{det}\left(I_{n-1}-t R D\right),
$$

and the conclusion follows from Lemmas 4.10, 4.11 and Theorem 4.13 ,

Remark 5.2. Let $x_{0} \in \partial \Omega$. From (4.24) we have that

$$
W\left(x_{0}\right) D \rho\left(\nu\left(x_{0}\right)\right)=-D^{2} \rho\left(D d_{\Omega}\left(x_{0}\right)\right) D^{2} d_{\Omega}\left(x_{0}\right) D \rho\left(\nu\left(x_{0}\right)\right)=0
$$

that is, $D \rho\left(\nu\left(x_{0}\right)\right)$ (and hence $D \rho\left(D d_{\Omega}\left(x_{0}\right)\right)$, by (4.20) $)$ is an eigenvector of $W\left(x_{0}\right)$ with corresponding eigenvalue zero. Since $\left\langle D \rho\left(\nu\left(x_{0}\right)\right), \nu\left(x_{0}\right)\right\rangle=\rho\left(\nu\left(x_{0}\right)\right) \neq 0$, then $D \rho\left(\nu\left(x_{0}\right)\right) \notin T_{x_{0}}$ is not an eigenvector for $\bar{W}\left(x_{0}\right)$. On the other hand, from Lemma 5.1 we deduce that a number $\kappa \neq 0$ is an eigenvalue of $W\left(x_{0}\right)$ if and only if it is an eigenvalue of $\bar{W}\left(x_{0}\right)$.

Remark 5.3. Although the matrix $\bar{W}\left(x_{0}\right)$ is not in general symmetric, its eigenvalues are real numbers (and so its eigenvectors are real). This property easily follows from (5.4), and the fact that $R$ and $D$ are symmetric matrices. Moreover, since $\bar{W}(x)$ is a continuous matrix-valued function on $\partial \Omega$, its eigenvalues are continuous real functions on $\partial \Omega$.

Lemma 5.4. Let $x \in \bar{\Omega}$ and let $x_{0} \in \Pi(x)$. If $\kappa$ is an eigenvalue of $\bar{W}\left(x_{0}\right)$, then $\kappa d_{\Omega}(x) \leq 1$. Furthermore, if $x \in \bar{\Omega} \backslash \mathcal{R}$, then $\kappa d_{\Omega}(x)<1$.

Proof. If $\kappa \leq 0$, then there is nothing to prove. Assume that $\kappa>0$. Then $\kappa$ is an eigenvalue of $\bar{W}\left(x_{0}\right)$ if and only if

$$
\operatorname{det}\left[\kappa I_{n-1}-\bar{W}\left(x_{0}\right)\right]=\kappa^{n-1} \operatorname{det}\left[I_{n-1}-\frac{1}{\kappa} \bar{W}\left(x_{0}\right)\right]=0 .
$$


From Lemma 5.1 this can happen only if $1 / \kappa \geq l\left(x_{0}\right)$, that is, if $\kappa l\left(x_{0}\right) \leq 1$. Since $l\left(x_{0}\right) \geq d_{\Omega}(x)$, this proves that $\kappa d_{\Omega}(x) \leq 1$. Furthermore, if $x \in \bar{\Omega} \backslash \mathcal{R}$, then $l\left(x_{0}\right)>d_{\Omega}(x)$, so that $\kappa d_{\Omega}(x)<1$.

The eigenvalues of $\bar{W}\left(x_{0}\right)$ have a significant geometric interpretation (see Remarks 5.7 and 5.8).

Definition 5.5 ( $\rho$-curvatures). Let $x_{0} \in \partial \Omega$. The principal $\rho$-curvatures of $\partial \Omega$ at $x_{0}$, with respect to the Minkowski norm $d_{\Omega}$, are the eigenvalues $\tilde{\kappa}_{1}\left(x_{0}\right) \leq \cdots \leq$ $\tilde{\kappa}_{n-1}\left(x_{0}\right)$ of $\bar{W}\left(x_{0}\right)$. The corresponding eigenvectors are the principal $\rho$-directions of $\partial \Omega$ at $x_{0}$.

Example 5.6 (Euclidean distance). Let $K=\bar{B}_{1}(0)$, so that $\rho(\xi)=|\xi|$. In this case, $d_{\Omega}$ coincides with the Euclidean distance $d_{\Omega}^{E}$ from $\partial \Omega$. Let $x_{0} \in \partial \Omega$ and consider the principal coordinate system at $x_{0}$. Since

$$
D \rho(\xi)=\frac{\xi}{|\xi|}, \quad D^{2} \rho(\xi)=\frac{1}{|\xi|} I-\frac{1}{|\xi|^{3}} \xi \otimes \xi, \quad \xi \neq 0,
$$

from Lemma 4.18 we recover $W\left(x_{0}\right)=\operatorname{diag}\left(\kappa_{1}, \ldots, \kappa_{n-1}, 0\right)=-D^{2} d_{\Omega}^{E}\left(x_{0}\right)$ and $\bar{W}\left(x_{0}\right)=\operatorname{diag}\left(\kappa_{1}, \ldots, \kappa_{n-1}\right)$. Hence the principal $\rho$-curvatures and the principal $\rho$-directions correspond respectively to the principal curvatures and the principal directions of $\partial \Omega$ at $x_{0}$.

Remark 5.7 (Normal curvatures). Definition 5.5 is motivated by the following construction. Let $x_{0} \in \partial \Omega$ and consider the principal coordinate system at $x_{0}$. Let $X: \mathcal{U} \rightarrow \mathbb{R}^{n}$ be a standard parameterization of $\partial \Omega$ in a neighborhood of $x_{0}$ satisfying (4.11). Let $v \in \mathbb{R}^{n-1}, v \neq 0$, and consider the curve on $\partial \Omega$,

$$
x(t)=X(t v), \quad t \in I,
$$

where $I=\left(-t_{0}, t_{0}\right)$ and $t_{0}>0$ is chosen such that $t v \in \mathcal{U}$ for every $t \in I$. Let us denote by $V$ the plane generated by $v$ and $\nu=\nu\left(x_{0}\right)=e_{n}$. For $r>0$, let $z_{r}=x_{0}+r D \rho(\nu)$ and let $K_{r}=z_{r}-r K^{0}$, so that $x_{0} \in K_{r} \cap \partial \Omega$. We would like to determine $r>0$ in such a way that the section $K_{r} \cap V$ has a contact of order two with the curve $x(t)$ at $x_{0}$. Such a value of $r=r(v)$ will be the radius of curvature of the curve $x(t)$ at $x_{0}$ with respect to the Minkowski distance; hence $\tilde{\kappa}(v)=1 / r(v)$ will be the curvature of $x(t)$ at $x_{0}$, that is, the normal curvature of $\partial \Omega$ at $x_{0}$ in the direction of $v$ with respect to the Minkowski distance.

For $t \in I$, the distance (with respect to the Minkowski norm) from $x(t)$ to the section $K_{r} \cap V$ can be estimated by $\left|\rho^{0}\left(z_{r}-x(t)\right)-r\right|$. The condition of a contact of second order becomes

$$
\begin{aligned}
0 & =\left.\frac{d^{2}}{d t^{2}} \rho^{0}\left(z_{r}-x(t)\right)\right|_{t=0} \\
& =\left\langle D^{2} \rho^{0}\left(z_{r}-x_{0}\right) \dot{x}(0), \dot{x}(0)\right\rangle+\left\langle D \rho^{0}\left(z_{r}-x_{0}\right), \ddot{x}(0)\right\rangle .
\end{aligned}
$$

Now, $z_{r}-x_{0}=r D \rho(\nu)$, so that from homogeneity and Lemma 2.2 we get

$$
D^{2} \rho^{0}\left(z_{r}-x_{0}\right)=\frac{1}{r} D^{2} \rho^{0}(D \rho(\nu)), \quad D \rho^{0}\left(z_{r}-x_{0}\right)=D \rho^{0}(D \rho(\nu))=\frac{\nu}{\rho(\nu)} .
$$

On the other hand, using (4.8) and (4.11) we get

$$
\dot{x}(0)=(v, 0), \quad \ddot{x}(0)=\left(0, \sum_{i=1}^{n-1} \kappa_{i} v_{i}^{2}\right) ;
$$


hence (5.5) becomes

$$
\frac{1}{r} \sum_{i, j=1}^{n-1}\left\langle D^{2} \rho^{0}(D \rho(\nu)) e_{j}, e_{i}\right\rangle v_{i} v_{j}+\frac{1}{\rho(\nu)} \sum_{i=1}^{n-1} \kappa_{i} v_{i}^{2}=0 .
$$

Recalling the definitions of the matrices $H$ and $D$ in (4.16) and (4.15) respectively, we conclude that

$$
\frac{1}{r}\langle H v, v\rangle-\frac{1}{\rho(\nu)}\langle D v, v\rangle=0 .
$$

Let us define $\tilde{\kappa}=\tilde{\kappa}(v)=1 / r$. The identity above can we rewritten as

$$
\tilde{\kappa}(v)\langle\rho(\nu) H v, v\rangle-\langle D v, v\rangle=0 .
$$

By construction, the quantity $\tilde{\kappa}(v)$ is the normal curvature of $\partial \Omega$ at $x_{0}$ along the direction $v$, with respect to the Minkowski norm. By analogy with what happens in the Riemannian case, the principal $\rho$-curvatures can be defined as the invariants of the pair of quadratic forms $D$ and $\rho(\nu) H$, that is, the numbers $\lambda \in \mathbb{R}$ that satisfy the characteristic equation

$$
\operatorname{det}(D-\lambda \rho(\nu) H)=0 .
$$

On the other hand, from (4.17) we have that $(\rho(\nu) H)^{-1}=R$, where $R$ is the matrix defined in (4.15); hence the invariants are the solutions of $\operatorname{det}\left(R D-\lambda I_{n-1}\right)=0$. Recalling (5.4), these are the solutions to

$$
\operatorname{det}\left(\bar{W}\left(x_{0}\right)-\lambda I_{n-1}\right)=0,
$$

that is, the eigenvalues of $\bar{W}\left(x_{0}\right)$. Moreover, if $\tilde{\kappa}_{1}\left(x_{0}\right) \leq \cdots \leq \tilde{\kappa}_{n-1}\left(x_{0}\right)$ are the eigenvalues of $\bar{W}\left(x_{0}\right)$ and $v_{1}, \ldots, v_{n-1}$ are the corresponding eigenvectors, then it is straightforward to check that $\tilde{\kappa}_{i}\left(x_{0}\right)=\tilde{\kappa}\left(v_{i}\right)$ for every $i=1, \ldots, n-1$.

Remark 5.8. Let $x_{0} \in \partial \Omega$ and let $\tilde{\kappa}_{1}\left(x_{0}\right) \leq \cdots \leq \tilde{\kappa}_{n-1}\left(x_{0}\right)$ be the principal $\rho$ curvatures of $\partial \Omega$ at $x_{0}$, that is, the eigenvalues of $\bar{W}\left(x_{0}\right)$. We claim that

$$
\tilde{\kappa}_{1}\left(x_{0}\right) \leq \tilde{\kappa}(v) \leq \tilde{\kappa}_{n-1}\left(x_{0}\right) \quad \forall v \in U_{x_{0}}:=\left\{w \in T_{x_{0}} \Omega ;\|w\|=1\right\},
$$

where $\tilde{\kappa}(v)$ is the normal curvature of $\partial \Omega$ at $x_{0}$ along the direction $v$, defined in (5.7), and $U_{x_{0}}$ is the unit tangent bundle of $\partial \Omega$ at $x_{0}$. More precisely, we claim that

$$
\tilde{\kappa}_{1}\left(x_{0}\right)=\min _{v \in U_{x_{0}}} \tilde{\kappa}(v), \quad \tilde{\kappa}_{n-1}\left(x_{0}\right)=\max _{v \in U_{x_{0}}} \tilde{\kappa}(v) .
$$

Namely, in the principal coordinate system at $x_{0}, \tilde{\kappa}: \mathbb{R}^{n-1} \backslash\{0\} \rightarrow \mathbb{R}$ is a continuous function on $S^{n-2}$; hence it admits a maximum and a minimum on $S^{n-2}$. Let $\bar{v} \in S^{n-2}$ be a maximum point. Since $\tilde{\kappa}$ is positively 0 -homogeneous (see (5.7)), then $\bar{v}$ is also a maximum point of $\tilde{\kappa}$ in the open set $\mathbb{R}^{n} \backslash\{0\}$, so that $D \tilde{\kappa}(\bar{v})=0$. Differentiating (5.7) and plugging in this identity we obtain

$$
\tilde{\kappa}(\bar{v}) \rho(\nu) H \bar{v}-D \bar{v}=0 ;
$$

that is, $\operatorname{det}(\tilde{\kappa}(\bar{v}) \rho(\nu) H-D)=0$. Thus $\tilde{\kappa}(\bar{v})$ is an invariant of the pair of quadratic forms $D$ and $\rho(\nu) H$, and hence an eigenvalue of $\bar{W}\left(x_{0}\right)$. Finally, $\tilde{\kappa}(\bar{v})$ being the maximum of $\tilde{\kappa}$ on $S^{n-2}$, we conclude that $\tilde{\kappa}(\bar{v})=\tilde{\kappa}_{n-1}\left(x_{0}\right)$. Reasoning as above, if $\bar{w}$ is a minimum point of $\tilde{\kappa}$ on $S^{n-2}$, we deduce that $\tilde{\kappa}(\bar{w})=\tilde{\kappa}_{1}\left(x_{0}\right)$. 
Remark 5.9 (Anisotropic Weingarten map). We can also give another interpretation of $\rho$-curvatures (see $[2, \S 2.3]$ ). We recall that, by definition, the principal curvatures of a smooth manifold $M$ at a given point $x \in M$ are the eigenvalues of the Weingarten map $L_{x}=-d \nu_{x}: T_{x} M \rightarrow T_{x} M$, where $\nu(x)$ is the normal to $M$ at $x$. In our setting, the inward "normal" to $\partial \Omega$ at $x$ is given by the vector $p(x):=D \rho(\nu(x))$ (see Proposition 4.4 and Remark 4.5) $)$. Hence the Weingarten map, in the Minkowskian setting, should be $L_{x}^{M}=-d p_{x}: T_{x} \Omega \rightarrow T_{x} \Omega$. It is readily seen that $L_{x}^{M}$ coincides with the map $\bar{W}(x)$ defined in (5.2). Hence, the principal curvatures at $x$ with respect to the Minkowski distance are the eigenvalues of the anisotropic Weingarten map $\bar{W}(x)$.

Remark 5.10 (Curvatures in Finsler geometry). If $\rho^{0}$ is of class $C^{\infty}$, then the principal $\rho$-curvatures coincide with the notion of curvature in the setting of Finsler geometry as defined in [24].

\section{Regularity of $\bar{\Sigma}$}

In this section we investigate the structure of the set $\bar{\Sigma}$. We shall prove that $\bar{\Sigma}=\mathcal{R}$, and that $d_{\Omega}$ is a function of class $C^{2}$ in the whole $\bar{\Omega} \backslash \bar{\Sigma}$. Finally we propose a regularity result for the set $\bar{\Sigma}$. The main tool for the proof of these results will be a complete description of the set $\bar{\Sigma} \backslash \Sigma$.

Definition 6.1 (Focal points). Let us define the map

$$
\Phi(x, t)=x+t D \rho(\nu(x)), \quad x \in \partial \Omega, t \in \mathbb{R} .
$$

For fixed $x_{0} \in \partial \Omega$, we say that $z=x_{0}+t_{0} D \rho\left(\nu\left(x_{0}\right)\right)$ is a focal point of $x_{0}$ if the map $\Phi$ is singular at $\left(x_{0}, t_{0}\right)$, that is, if $\operatorname{det} D \Phi\left(x_{0}, t_{0}\right)=0$.

In the setting of Riemannian geometry, the point $z$ in the above definition would be called a focal point of $\partial \Omega$ along the geodesic $t \mapsto \Phi\left(x_{0}, t\right), t \geq 0$, normal to $\partial \Omega$ at $x_{0}$ (see e.g. [22]).

Remark 6.2. Let $Y: \mathcal{U} \rightarrow \mathbb{R}^{n}, \mathcal{U} \subset \mathbb{R}^{n-1}$ open, be a local parameterization of $\partial \Omega$ in a neighborhood of $x_{0}$, such that $Y(0)=x_{0}$. The fact that $z=x_{0}+t_{0} D \rho\left(\nu\left(x_{0}\right)\right)$ is a focal point amounts to saying that the differential of the map $\Psi(y, t):=Y(y)+$ $t D \rho(\nu(Y(y)))$ at $\left(0, t_{0}\right)$ does not have maximal rank, that is, $\operatorname{det} D \Psi\left(0, t_{0}\right)=0$. Recalling (4.13), (4.14) and (5.4), we have

$$
\operatorname{det} D \Psi\left(0, t_{0}\right)=0 \quad \Longleftrightarrow \quad \operatorname{det}\left(I_{n-1}-t_{0} \bar{W}\left(x_{0}\right)\right)=0 ;
$$

hence the focal points of $x_{0} \in \partial \Omega$ are $\Phi\left(x_{0}, 1 / \tilde{\kappa}_{i}\left(x_{0}\right)\right)$, if $\tilde{\kappa}_{i}\left(x_{0}\right) \neq 0, i=1, \ldots, n-1$. Moreover, by Theorem 4.13, $l\left(x_{0}\right) \leq 1 / \tilde{\kappa}_{n-1}\left(x_{0}\right)$.

Definition 6.3 (Optimal focal points). Let $x_{0} \in \partial \Omega$. If $\tilde{\kappa}_{n-1}\left(x_{0}\right)>0$, we call $z_{0}=x_{0}+1 / \tilde{\kappa}_{n-1}\left(x_{0}\right) D \rho\left(\nu\left(x_{0}\right)\right)$ the first focal point of $x_{0}$. If, in addition, $z_{0} \in \bar{\Sigma}$, we call $z_{0}$ the optimal focal point of $x_{0}$. We denote by $\Gamma$ the set of optimal focal points of the boundary points of $\Omega$.

In the following proposition we prove that $\bar{\Sigma}=\Sigma \cup \Gamma$.

Proposition 6.4. If $x \in \bar{\Sigma} \backslash \Sigma$, then $\tilde{\kappa}_{n-1}\left(x_{0}\right) d_{\Omega}(x)=1$, where $x_{0}$ is the unique projection of $x$ on $\partial \Omega$. 
Proof. Let $x \in \bar{\Sigma} \backslash \Sigma$, and let $\left(x_{k}\right) \subset \Sigma$ be a sequence converging to $x$. For every $k \in \mathbb{N}$, let $y_{k}, z_{k} \in \partial \Omega$ be two distinct points in $\Pi\left(x_{k}\right)$, so that

$$
x_{k}=y_{k}+d_{\Omega}\left(x_{k}\right) D \rho\left(D d_{\Omega}\left(y_{k}\right)\right)=z_{k}+d_{\Omega}\left(x_{k}\right) D \rho\left(D d_{\Omega}\left(z_{k}\right)\right),
$$

and let

$$
\xi_{k}=\frac{y_{k}-z_{k}}{\left|y_{k}-z_{k}\right|} .
$$

Since $\xi_{k} \in S^{n-1}$ for every $k$, we can extract a subsequence (which we do not relabel) converging to a point $\xi \in S^{n-1}$. From Lemma 3.4 we have that $\left(y_{k}\right)_{k}$ and $\left(z_{k}\right)_{k}$ converge to $x_{0}$. From (6.2) we get

$$
\frac{y_{k}-z_{k}}{\left|y_{k}-z_{k}\right|}+d_{\Omega}\left(x_{k}\right) \frac{D \rho\left(D d_{\Omega}\left(y_{k}\right)\right)-D \rho\left(D d_{\Omega}\left(z_{k}\right)\right)}{\left|y_{k}-z_{k}\right|}=0 \quad \forall k \in \mathbb{N} .
$$

Since $d_{\Omega}$ is of class $C^{2}$ in a neighborhood of $\partial \Omega$ and $\rho$ is of class $C^{2}$ in $\mathbb{R}^{n}$ $\{0\}$, passing to the limit we get $\xi+d_{\Omega}(x) D^{2} \rho\left(D d_{\Omega}\left(x_{0}\right)\right) D^{2} d_{\Omega}\left(x_{0}\right) \xi=0$, that is, $d_{\Omega}(x) W\left(x_{0}\right) \xi=\xi$. Hence $1 / d_{\Omega}(x)$ is a nonzero eigenvalue of $W\left(x_{0}\right)$. From Remark [5.2, we conclude that $1 / d_{\Omega}(x)$ is an eigenvalue of $\bar{W}\left(x_{0}\right)$, that is, $1 / d_{\Omega}(x)=$ $\tilde{\kappa}_{j}\left(x_{0}\right)$ for some $j \in\{1, \ldots, n-1\}$.

Finally, let us prove that $\tilde{\kappa}_{j}\left(x_{0}\right)=\tilde{\kappa}_{n-1}\left(x_{0}\right)$. Assume, by contradiction, that $\tilde{\kappa}_{j}\left(x_{0}\right)<\tilde{\kappa}_{n-1}\left(x_{0}\right)$. Then $1 / \tilde{\kappa}_{n-1}\left(x_{0}\right)<1 / \tilde{\kappa}_{j}\left(x_{0}\right)=d_{\Omega}\left(x_{0}\right) \leq l\left(x_{0}\right)$, a contradiction (see Remark 6.2).

Proposition 6.5. Let $x \in \Omega$ and $x_{0} \in \Pi(x)$. Then $\bar{\Sigma} \cap\left(x_{0}, x\right)=\emptyset$.

Proof. By Proposition 3.3(iii) we already know that $\Sigma \cap\left(x_{0}, x\right)=\emptyset$. Moreover, from Lemma 5.4 we have that $\tilde{\kappa}_{n-1}\left(x_{0}\right) d_{\Omega}(x) \leq 1$; hence the strict inequality $\tilde{\kappa}_{n-1}\left(x_{0}\right) d_{\Omega}(z)<1$ holds for every $z \in\left(x_{0}, x\right)$. This implies, by Proposition 6.4, that $(\bar{\Sigma} \backslash \Sigma) \cap\left(x_{0}, x\right)=\emptyset$, concluding the proof.

Corollary 6.6. For every $x_{0} \in \partial \Omega$,

$$
l\left(x_{0}\right)=\min \left\{t \geq 0 ; x_{0}+t D \rho\left(\nu\left(x_{0}\right)\right) \in \bar{\Sigma}\right\} .
$$

Proof. Let us denote by $\lambda\left(x_{0}\right)$ the right-hand side of (6.3). Notice that the point $z_{0}=x_{0}+l\left(x_{0}\right) D \rho\left(\nu\left(x_{0}\right)\right)$ belongs to the ridge set; then, by Remark 4.8, $z_{0} \in \bar{\Sigma}$. Hence we have $\lambda\left(x_{0}\right) \leq l\left(x_{0}\right)$. Assume by contradiction that the strict inequality holds. Then, if we set $z(t):=x_{0}+t D \rho\left(\nu\left(x_{0}\right)\right)$, for $\bar{t} \in\left(\lambda\left(x_{0}\right), l\left(x_{0}\right)\right)$ we would have $x_{0} \in \Pi(z(\bar{t}))$ and $z\left(\lambda\left(x_{0}\right)\right) \in\left(x_{0}, z(\bar{t})\right) \cap \bar{\Sigma}$, in contradiction with Proposition 6.5.

Theorem 6.7. The function $l$, defined in (3.11), and the function $\tau: \bar{\Omega} \rightarrow \mathbb{R}$ defined by

$$
\tau(x)= \begin{cases}\min \left\{t \geq 0 ; x+t D \rho\left(D d_{\Omega}(x)\right) \in \bar{\Sigma}\right\}, & \text { if } x \in \bar{\Omega} \backslash \bar{\Sigma} \\ 0, & \text { if } x \in \bar{\Sigma}\end{cases}
$$

(and called the normal distance to the cut locus of $x$ ) are continuous in $\bar{\Omega}$.

Proof. It is easy to check that, if $x \in \bar{\Omega}$ and $x_{0} \in \Pi(x)$, then $\tau(x)=\tau\left(x_{0}\right)-d_{\Omega}(x)$. Since, by Corollary 6.6, $\tau\left(x_{0}\right)=l\left(x_{0}\right)=l(x)$, we get $\tau(x)=l(x)-d_{\Omega}(x)$ for every $x \in \bar{\Omega}$. From Proposition 3.10 the function $l$ is upper semicontinuous in $\Omega$; hence the upper semicontinuity of $\tau$ follows from the continuity of $d_{\Omega}$. On the other hand, the lower semicontinuity of $\tau$ at a point $x \in \bar{\Sigma}$ is trivial. Hence it remains to prove that $\tau$ is lower semicontinuous in $x \in \bar{\Omega} \backslash \bar{\Sigma}$. Let $\left(x_{k}\right)_{k}$ be a sequence 
in $\bar{\Omega}$ converging to $x$. We can assume that $x_{k} \in \bar{\Omega} \backslash \bar{\Sigma}$; hence, by definition of $\tau$, $x_{k}+\tau\left(x_{k}\right) D \rho\left(D d_{\Omega}\left(x_{k}\right)\right) \in \bar{\Sigma}$. If $\tau_{0}:=\liminf _{k} \tau\left(x_{k}\right)$, passing to the limit we get $x+\tau_{0} D \rho\left(D d_{\Omega}(x)\right) \in \bar{\Sigma}$, that is, $\tau(x) \leq \tau_{0}$.

Corollary 6.8. The ridge set $\mathcal{R}$ is closed and $\mathcal{R}=\bar{\Sigma}$.

Proof. We recall that $\mathcal{R}=\left\{x \in \Omega ; l(x)=d_{\Omega}(x)\right\}$. Since, by Lemma 4.1, $\overline{\mathcal{R}} \subset \Omega$, the fact that $\mathcal{R}$ is closed follows from the continuity of $l$ and $d_{\Omega}$. The equality $\mathcal{R}=\bar{\Sigma}$ now follows from the inclusions $\Sigma \subseteq \mathcal{R} \subseteq \bar{\Sigma}$.

Corollary 6.9. The set $\bar{\Sigma}$ has vanishing Lebesgue measure.

Proof. The proof follows from Corollaries 4.15 and 6.8 ,

We can now prove a regularity result for $d_{\Omega}$, which extends Theorem 4.16 to $\bar{\Omega} \backslash \bar{\Sigma}$.

Theorem 6.10 (Regularity of $d_{\Omega}$ in $\bar{\Omega} \backslash \bar{\Sigma}$ ). The function $d_{\Omega}$ is of class $C^{2}$ in $\bar{\Omega} \backslash \bar{\Sigma}$. If $\Omega$ is of class $C^{k, \alpha}$ and $\rho \in C^{k, \alpha}\left(\mathbb{R}^{n} \backslash\{0\}\right)$, for some $k \geq 2$ and $\alpha \in[0,1]$, then $d_{\Omega}$ is of class $C^{k, \alpha}$ in $\bar{\Omega} \backslash \bar{\Sigma}$.

Proof. It is enough to prove the result in $\Omega \backslash \bar{\Sigma}$, since the regularity of $d_{\Omega}$ near $\partial \Omega$ was already proved in Theorem 4.16 .

For fixed $z_{0} \in \Omega \backslash \bar{\Sigma}$, we have to show that $d_{\Omega}$ is of class $C^{2}$ in a neighborhood of $z_{0}$. Let $\Pi\left(z_{0}\right)=\left\{x_{0}\right\}$, and let $Y: \mathcal{U} \rightarrow \mathbb{R}^{n}, \mathcal{U} \subset \mathbb{R}^{n-1}$ open, be a local parameterization of $\partial \Omega$ in a neighborhood of $x_{0}$. Let $\Psi$ be the map defined in (4.12), and let $t_{0}=d_{\Omega}\left(z_{0}\right)$. By Corollary 6.8, $z_{0}$ does not belong to $\mathcal{R}$, that is, $d_{\Omega}\left(z_{0}\right)<l\left(z_{0}\right)=l\left(x_{0}\right)$. From Theorem 4.13 we have that $\operatorname{det} D \Psi\left(x_{0}, t_{0}\right)>0$; hence from the inverse mapping theorem it follows that there exists a neighborhood $V$ of $z_{0}$ such that the map $y=y(x)$ is of class $C^{1}(V)$. On the other hand, from Lemma 4.3. $D d_{\Omega}(x)=N(y(x)) / \rho(N(y(x))), x \in V$, where $N=\nu \circ Y$; hence we conclude that $D d_{\Omega} \in C^{1}(V)$, that is, $d_{\Omega} \in C^{2}(V)$.

The last part of the proposition follows from the fact that, if $\Omega$ is of class $C^{k, \alpha}$ and $\rho \in C^{k, \alpha}\left(\mathbb{R}^{n} \backslash\{0\}\right)$, then $\Psi \in C^{k-1, \alpha}(\mathcal{U} \times \mathbb{R})$.

In the literature, the set

$$
\operatorname{Cut}(\Omega)=\left\{\Phi\left(x_{0}, l\left(x_{0}\right)\right)=x_{0}+l\left(x_{0}\right) D \rho\left(\nu\left(x_{0}\right)\right), x_{0} \in \partial \Omega\right\}
$$

is called the cut locus of $\Omega$. From (6.3), Corollary 6.8, and Proposition 6.4 it follows that

$$
\operatorname{Cut}(\Omega)=\mathcal{R}=\bar{\Sigma}=\Sigma \cup \Gamma \text {. }
$$

The regularities of the singular set $\Sigma$ and of the cut locus $\bar{\Sigma}$ have been extensively studied. For what concerns $\Sigma$, Alberti [1] has proved that it is $C^{2}$-rectifiable. We recall that a subset of $\mathbb{R}^{n}$ is $C^{k}$-rectifiable, $k \in \mathbb{N}$, if it can be covered by a countable family of embedded $C^{k}$ manifolds of dimension $n-1$, with the exception of a set of vanishing $\mathcal{H}^{n-1}$ measure. On the other hand, in general, the cut locus $\bar{\Sigma}$ is not rectifiable. Namely, Mantegazza and Mennucci 17] have exhibited a set $\Omega \subset \mathbb{R}^{2}$ of class $C^{1,1}$ such that the singular set $\Sigma$ corresponding to the Euclidean distance $d_{\Omega}^{E}$ has closure $\bar{\Sigma}$ with positive Lebesgue measure. In the same paper the authors have proven that, if $\Omega$ is an open subset of class $C^{r}$, with $r \geq 3$, of a smooth, connected and complete Riemannian manifold without boundary, then $\operatorname{Cut}(\Omega)$ is $(r-2)$-rectifiable. In the same setting, Itoh and Tanaka [14] have proven that, if $\Omega$ 
is of class $C^{\infty}$, then the function $l$ is Lipschitz continuous on $\partial \Omega$. Recently, Li and Nirenberg [15] have refined this result, extending it to the case of Finsler manifolds. Adapting their result to our setting, they have proved that, if $\Omega$ is of class $C^{2,1}$ and $\rho$ is of class $C^{\infty}$, then $l$ is Lipschitz continuous on $\partial \Omega$ (see [15], Theorem 1.5). As a straightforward consequence of the fact that $l$ is Lipschitz continuous on $\partial \Omega$, one has that the cut locus $\bar{\Sigma}$ has finite $\mathcal{H}^{n-1}$ measure and it is $C^{1}$-rectifiable. Namely, if $L$ denotes the Lipschitz constant of $l$ in $\partial \Omega$, we have $\mathcal{H}^{n-1}(\bar{\Sigma}) \leq L \mathcal{H}^{n-1}(\partial \Omega)<+\infty$. Other results in this direction are proved in [18, again in the setting of Finsler manifolds. We mention also [7, 8] for further regularity results for Hamilton-Jacobi equations, and [19] for rectifiability results of $\bar{\Sigma}$ from an optimal control theory viewpoint.

In the following theorem we shall prove a $C^{0, \alpha}$-rectifiability result of the cut locus in the Minkowskian setting. For what concerns the regularity of the cut locus, our result is finer than the ones given in [15, 18, that, on the other hand, deal with a general Finsler manifold.

Theorem 6.11 (Regularity of $\bar{\Sigma}$ ). Assume that, for some $\alpha \in[0,1], \Omega$ is of class $C^{2, \alpha}$, and $\rho, \rho^{0} \in C^{2, \alpha}\left(\mathbb{R}^{n} \backslash\{0\}\right)$. Then $\bar{\Sigma}$ can be covered by countably many graphs of functions of class $C^{0, \alpha}\left(\mathbb{R}^{n-1}\right)$, with the exception of a set of vanishing $\mathcal{H}^{n-1}$ measure. As a consequence, $\operatorname{dim}_{\mathcal{H}}(\bar{\Sigma}) \leq n-\alpha$, where $\operatorname{dim}_{\mathcal{H}}(\bar{\Sigma})$ denotes the Hausdorff dimension of $\bar{\Sigma}$.

Proof. Since $d_{\Omega}$ is semiconcave in $\Omega$, by Theorem 1 in [1] we have that $\Sigma$ is $C^{2}$ rectifiable. Hence it is enough to prove that the set of optimal focal points $\Gamma$ can be covered by countably many graphs of functions of class $C^{0, \alpha}$, defined on open subsets of $\mathbb{R}^{n-1}\left(C^{0, \alpha}\right.$-rectifiable for short). Since $\Gamma \subseteq \mathcal{R}$ is contained in the set

$$
\Gamma_{0}=\left\{x_{0}+\frac{1}{\tilde{\kappa}_{n-1}\left(x_{0}\right)} D \rho\left(\nu\left(x_{0}\right)\right) ; x_{0} \in \partial \Omega \text { such that } \tilde{\kappa}_{n-1}\left(x_{0}\right) \geq 1 / L\right\}
$$

of all first focal points with distance from $\partial \Omega$ not exceeding the quantity $L:=$ $\max \{l(y) ; y \in \partial \Omega\}$, then it is enough to prove that $\Gamma_{0}$ is $C^{0, \alpha}$-rectifiable. Let us denote by $S_{0}$ the set

$$
S_{0}:=\left\{x_{0} \in \partial \Omega ; \tilde{\kappa}_{n-1}\left(x_{0}\right) \geq 1 / L\right\} .
$$

Let $\mathcal{U}_{1}, \ldots, \mathcal{U}_{N} \subset \mathbb{R}^{n-1}$ be open sets, and $Y_{k}: \mathcal{U}_{k} \rightarrow \mathbb{R}^{n}, k=1, \ldots, N$, maps of class $C^{2, \alpha}$ that parameterize $\partial \Omega$. For every $k=1, \ldots, N$, let $U_{k}:=\left\{y \in \mathcal{U}_{k} ; Y_{k}(y) \in S_{0}\right\}$. Then $S_{0}=\bigcup_{k=1}^{N} Y_{k}\left(U_{k}\right)$, and

$$
\Gamma_{0}=\bigcup_{k=1}^{N}\left\{\Psi_{k}\left(y, \frac{1}{\tilde{\kappa}_{n-1}\left(Y_{k}(y)\right)}\right) ; y \in U_{k}\right\}
$$

where, for every $k=1, \ldots, N$,

$$
\Psi_{k}(y, t):=Y_{k}(y)+t D \rho\left(\nu\left(Y_{k}(y)\right)\right), \quad(y, t) \in \mathcal{U}_{k} \times \mathbb{R} .
$$

Let us fix $k=1, \ldots, N$. We claim that the map $K:=\tilde{\kappa}_{n-1} \circ Y_{k}$ is of class $C^{0, \alpha}$ in $\mathcal{U}_{k}$. For every $y \in \mathcal{U}_{k}$ let us denote by $D(y)$ and $H(y)$ the matrices defined respectively in (4.15) and (4.16) in the point $x_{0}=Y_{k}(y)$. We recall that $D(y)$ and $H(y)$ are the restrictions respectively of $D^{2} d_{\Omega}\left(Y_{k}(y)\right)$ and $D^{2} \rho^{0}\left(D \rho\left(\nu\left(Y_{k}(y)\right)\right)\right)$ to the tangent space $T_{Y_{k}(y)} \Omega$. From Theorem 6.10, these functions are both of class $C^{0, \alpha}\left(\mathcal{U}_{k}\right)$. Thus, the matrix

$$
H_{0}(y):=\rho\left(\nu\left(Y_{k}(y)\right)\right) H(y), \quad y \in \mathcal{U}_{k},
$$


is of class $C^{0, \alpha}\left(\mathcal{U}_{k}\right)$. Furthermore, from Theorem 2.1(iv), the minimum eigenvalue of $H(y)$ is bounded from below by the minimum for $\xi \in \partial K$ of the radii of curvature of $\partial K$ at $\xi$ (which is strictly positive by the assumption $K \in C_{+}^{2}$ ). From (2.1) we conclude that there exists a constant $c>0$ such that

$$
\left\langle H_{0}(y) v, v\right\rangle \geq c\|v\|^{2}, \quad \forall y \in \mathcal{U}_{k}, v \in \mathbb{R}^{n-1} .
$$

From (5.9), $K(y)$ is characterized by

$$
K(y)=\max _{v \in S^{n-2}} \frac{\langle D(y) v, v\rangle}{\left\langle H_{0}(y) v, v\right\rangle}, \quad y \in \mathcal{U}_{k} .
$$

Now, let $y, y^{\prime} \in \mathcal{U}_{k}$, and denote by $v_{0}, v_{0}^{\prime} \in \mathbb{R}^{n-1}$ two vectors that realize the maximum in (6.7) for $K(y)$ and $K\left(y^{\prime}\right)$ respectively. Since $D, H_{0} \in C^{2, \alpha}\left(\mathcal{U}_{k}\right)$, from (6.6) we have that

$$
\begin{aligned}
K(y)-K\left(y^{\prime}\right) & \leq \frac{\left\langle D(y) v_{0}, v_{0}\right\rangle}{\left\langle H_{0}(y) v_{0}, v_{0}\right\rangle}-\frac{\left\langle D\left(y^{\prime}\right) v_{0}, v_{0}\right\rangle}{\left\langle H_{0}\left(y^{\prime}\right) v_{0}, v_{0}\right\rangle} \\
& \leq C \frac{\left\|H_{0}\left(y^{\prime}\right)\right\|+\left\|D\left(y^{\prime}\right)\right\|}{\left\langle H_{0}(y) v_{0}, v_{0}\right\rangle\left\langle H_{0}\left(y^{\prime}\right) v_{0}, v_{0}\right\rangle}\left|y-y^{\prime}\right|^{\alpha} \\
& \leq \frac{C S}{c^{2}}\left|y-y^{\prime}\right|^{\alpha},
\end{aligned}
$$

where $S:=\sup \left\{\left\|H_{0}(z)\right\|+\|D(z)\| ; z \in \mathcal{U}_{k}\right\}$. Exchanging the role of $y$ and $y^{\prime}$, we obtain $K\left(y^{\prime}\right)-K(y) \leq\left(C S / c^{2}\right)\left|y-y^{\prime}\right|^{\alpha}$; hence $K \in C^{0, \alpha}\left(\mathcal{U}_{k}\right)$.

Since $\Psi_{k} \in C^{1}\left(\mathcal{U}_{k} \times \mathbb{R}\right)$, with bounded derivatives in $\mathcal{U}_{k} \times[0, L]$ (see Lemma 4.10), it is Lipschitz continuous in $\mathcal{U}_{k} \times[0, L]$, say of rank $L_{k}$; hence

$$
\mathcal{H}^{n-\alpha}\left\{\Psi_{k}(y, 1 / K(y)) ; y \in U_{k}\right\} \leq L_{k}^{n-\alpha} \mathcal{H}^{n-\alpha}\left\{(y, 1 / K(y)) ; y \in U_{k}\right\} .
$$

On the other hand, $K: \mathcal{U}_{k} \subset \mathbb{R}^{n-1} \rightarrow \mathbb{R}$ being a positive function of class $C^{0, \alpha}$ on $\mathcal{U}_{k}$, with $K(y) \geq 1 / L$ for every $y \in U_{k}$, the function $1 / K$ is of class $C^{0, \alpha}$ on $U_{k}$. Thus the Hausdorff $(n-\alpha)$-dimensional measure of the graph of $1 / K$ on $U_{k}$ is finite. Since this fact holds for every $k=1, \ldots, N$, from the definition (6.5) of $\Gamma_{0}$ we conclude that $\mathcal{H}^{n-\alpha}\left(\Gamma_{0}\right)$ is finite.

\section{An APplication to PDEs}

This section is devoted to an application of the previous results to the analysis of PDEs arising from optimal transportation theory and shape optimization (see [5, 6]). The main tool is the following change of variables theorem, based on Lemma 4.10, Theorem 4.13 and the fact that $\bar{\Sigma}$ has vanishing Lebesgue measure.

Theorem 7.1 (Change of variables). For every $h \in L^{1}(\Omega)$,

$$
\begin{aligned}
& \int_{\Omega} h(x) d x=\int_{\partial \Omega} \rho(\nu(x))\left[\int_{0}^{l(x)} h(\Phi(x, t)) \operatorname{det}\left(I_{n-1}-t \bar{W}(x)\right) d t\right] d \mathcal{H}^{n-1}(x) \\
& =\int_{\partial \Omega} \rho(\nu(x))\left[\int_{0}^{l(x)} h(\Phi(x, t)) \prod_{i=1}^{n-1}\left(1-t \tilde{\kappa}_{i}(x)\right) d t\right] d \mathcal{H}^{n-1}(x)
\end{aligned}
$$

where $\Phi: \partial \Omega \times \mathbb{R} \rightarrow \mathbb{R}^{n}$ is the map defined by $\Phi(x, t)=x+t D \rho(\nu(x)),(x, t) \in$ $\partial \Omega \times \mathbb{R}$. 
Proof. Let $Y_{k}: \mathcal{U}_{k} \rightarrow \mathbb{R}^{n}, \mathcal{U}_{k} \subset \mathbb{R}^{n-1}$ open, $k=1, \ldots, N$, be local parameterizations of $\partial \Omega$ of class $C^{2}$, such that $\bigcup_{k=1}^{N} Y_{k}\left(\mathcal{U}_{k}\right)=\partial \Omega$. For every $k=1, \ldots, N$, let $\Psi_{k}: \mathcal{U}_{k} \times \mathbb{R} \rightarrow \mathbb{R}^{n}$ be the map

$$
\Psi_{k}(y, t)=Y_{k}(y)+t D \rho\left(\nu\left(Y_{k}(y)\right)\right), \quad(y, t) \in \mathcal{U}_{k} \times \mathbb{R} .
$$

From Lemma 4.10 and Theorem 4.13 we know that, for every $k=1, \ldots, N, \Psi_{k} \in$ $C^{1}\left(\mathcal{U}_{k} \times \mathbb{R}\right)$, and

$$
\operatorname{det} D \Psi_{k}(y, t)=\rho\left(\nu\left(Y_{k}(y)\right)\right) \sqrt{g^{k}(y)} \operatorname{det}\left[I_{n-1}-t \bar{W}\left(Y_{k}(y)\right)\right]>0
$$

for every $y \in \mathcal{U}_{k}$ and every $t \in\left[0, l\left(Y_{k}(y)\right)\right)$, where

$$
g^{k}(y)=\operatorname{det}\left(g_{i j}^{k}(y)\right), \quad g_{i j}^{k}(y)=\left\langle\frac{\partial Y_{k}}{\partial y_{i}}(y), \frac{\partial Y_{k}}{\partial y_{j}}(y)\right\rangle, \quad i, j=1, \ldots, n-1 .
$$

Let $p_{1}, \ldots, p_{N} \in \partial \Omega \rightarrow \mathbb{R}$ be a partition of unity of $\partial \Omega$ subordinate to $Y_{1}, \ldots, Y_{N}$; that is, for every $k=1, \ldots, N, p_{k}(x) \geq 0$ for every $x \in \partial \Omega, p_{k}$ has compact support contained in $Y_{k}\left(\mathcal{U}_{k}\right), p_{k} \circ Y_{k} \in C^{2}\left(\mathcal{U}_{k}\right)$, and $\sum_{k=1}^{N} p_{k}(x)=1$ for every $x \in \partial \Omega$. Moreover, for every $k=1, \ldots, N$, let us define the function

$$
q_{k}(x)= \begin{cases}p_{k}\left(x_{0}\right), & \text { if } x_{0} \in Y_{k}\left(\mathcal{U}_{k}\right) \text { and } x=\Phi\left(x_{0}, t\right) \text { for some } t \in\left[0, l\left(x_{0}\right)\right), \\ 0, & \text { otherwise }\end{cases}
$$

and the sets

$$
A_{k}=\left\{(y, t) ; y \in \mathcal{U}_{k}, t \in\left(0, l\left(Y_{k}(y)\right)\right)\right\}, \quad \Omega_{k}=\Psi_{k}\left(A_{k}\right) .
$$

By construction, it is readily seen that $q_{k}=0$ outside $\Omega_{k}$ for every $k$, and that $\sum_{k=1}^{N} q_{k}(x)=1$ for every $x \in \Omega \backslash \bar{\Sigma}$, hence, from Corollary 6.9 for almost every $x \in \Omega$. The first equality in (7.1) is obtained using, for every $k=1, \ldots, N$, the change of variables $\Psi_{k}$ on $A_{k}$, Fubini's theorem, and the area formula (see [12, $\S 3.3 .3$ and $\S 3.3 .4]$ ) as follows:

$$
\begin{aligned}
& \int_{\Omega} h(x) d x=\sum_{k=1}^{N} \int_{\Omega} q_{k}(x) h(x) d x=\sum_{k=1}^{N} \int_{\Omega_{k}} q_{k}(x) h(x) d x \\
& =\sum_{k=1}^{N} \int_{A_{k}} q_{k}\left(\Psi_{k}(y, t)\right) h\left(\Psi_{k}(y, t)\right) \operatorname{det} D \Psi_{k}(y, t) d t d y \\
& =\sum_{k=1}^{N} \int_{\mathcal{U}_{k}}\left[\int_{0}^{l\left(Y_{k}(y)\right)} q_{k}\left(Y_{k}(y)\right) h\left(\Psi_{k}(y, t)\right) \frac{\operatorname{det} D \Psi_{k}(y, t)}{\sqrt{g^{k}(y)}} d t\right] \sqrt{g^{k}(y)} d y \\
& =\sum_{k=1}^{N} \int_{Y_{k}\left(\mathcal{U}_{k}\right)} q_{k}(x)\left[\int_{0}^{l(x)} h(\Phi(x, t)) \rho(\nu(x)) D(x, t) d t\right] d \mathcal{H}^{n-1}(x) \\
& =\int_{\partial \Omega}\left[\int_{0}^{l(x)} h(\Phi(x, t)) \rho(\nu(x)) D(x, t) d t\right] d \mathcal{H}^{n-1}(x)
\end{aligned}
$$

where $D(x, t):=\operatorname{det}\left(I_{n-1}-t \bar{W}(x)\right)$. The second equality in (7.1) follows upon observing that $\tilde{\kappa}_{1}(x), \ldots, \tilde{\kappa}_{n-1}(x)$ are the eigenvalues of $\bar{W}(x)$ for every $x \in \partial \Omega$. 
Let us consider the following system of PDEs of Monge-Kantorovich type:

$$
\begin{cases}-\operatorname{div}(v D \rho(D u))=f & \text { in } \Omega, \\ \rho(D u) \leq 1 & \text { in } \Omega, \\ \rho(D u)=1 & \text { in }\{v>0\}\end{cases}
$$

where the source $f \geq 0$ is a continuous function in $\Omega$, complemented with the conditions

$$
\begin{cases}u \geq 0, v \geq 0 & \text { in } \Omega \\ u=0 & \text { on } \partial \Omega .\end{cases}
$$

The first equation in (7.3) has to be understood in the sense of distributions, whereas $u$ is a viscosity solution to $\rho(D u)=1$ in the set $\{v>0\}$. We look for a solution $(u, v)$ to (7.3)-(7.4) in the class of continuous and nonnegative functions.

Since $d_{\Omega}$ is a viscosity solution of $\rho(D u)=1$ in $\Omega, u=0$ on $\partial \Omega$, it is clear that $d_{\Omega}$ is a viscosity solution, vanishing on $\partial \Omega$, of the equation $\rho\left(D d_{\Omega}\right)=1$ in $\{v>0\}$, for every fixed continuous function $v \in C(\Omega)$. Then it is enough to prove that the equation

$$
-\operatorname{div}\left(v(x) D \rho\left(D d_{\Omega}(x)\right)\right)=f(x) \quad \text { in } \Omega
$$

has a continuous nonnegative solution. More precisely, we are interested in finding a function $v \in C(\Omega)$ satisfying

$$
\int_{\Omega} v(x)\left\langle D \rho\left(D d_{\Omega}(x)\right), D \varphi(x)\right\rangle d x=\int_{\Omega} f(x) \varphi(x) d x
$$

for every $\varphi$ belonging to the set $C_{c}^{\infty}(\Omega)$ of functions of class $C^{\infty}(\Omega)$ with compact support in $\Omega$.

In order to write explicitly a solution $v$ of (7.5), it will be convenient to extend the functions $\Phi$, defined on $\partial \Omega$ in Theorem 7.1 , and $\tilde{\kappa}_{i}, i=1, \ldots, n-1$, defined on $\partial \Omega$ in Definition 5.5. to $\bar{\Omega} \backslash \bar{\Sigma}$ in the following way. If $x \in \bar{\Omega} \backslash \bar{\Sigma}$ and $\Pi(x)=\left\{x_{0}\right\}$, we set

$$
\Phi(x, t):=x_{0}+\left(d_{\Omega}(x)+t\right) D \rho\left(\nu\left(x_{0}\right)\right), \quad \tilde{\kappa}_{i}(x):=\tilde{\kappa}_{i}\left(x_{0}\right), \quad i=1, \ldots, n-1 .
$$

Since, from Lemma 3.4, the map which associates to every point $x \in \bar{\Omega} \backslash \bar{\Sigma}$ its unique projection on $\partial \Omega$ is continuous in $\bar{\Omega} \backslash \bar{\Sigma}$, we conclude that the maps $\tilde{\kappa}_{i}$, $i=1, \ldots, n-1$, which are continuous on $\partial \Omega$ (see Remark 5.3) are also continuous in $\bar{\Omega} \backslash \bar{\Sigma}$, and the map $\Phi$ in continuous in $(\bar{\Omega} \backslash \bar{\Sigma}) \times \mathbb{R}$.

The remaining part of this section will be devoted to the proof of the following theorem.

Theorem 7.2. Let $f \in C(\Omega), f \geq 0$. Then the function

$$
v_{f}(x)= \begin{cases}\int_{0}^{\tau(x)} f(\Phi(x, t)) \prod_{i=1}^{n-1} \frac{1-\left(d_{\Omega}(x)+t\right) \tilde{\kappa}_{i}(x)}{1-d_{\Omega}(x) \tilde{\kappa}_{i}(x)} d t & \text { if } x \in \bar{\Omega} \backslash \bar{\Sigma}, \\ 0, & \text { if } x \in \bar{\Sigma},\end{cases}
$$

is continuous in $\Omega$ and satisfies (17.6) for every $\varphi \in C_{c}^{\infty}(\Omega)$. Here $\tau$ is the distance to the cut locus defined in (6.4).

Before proving Theorem 7.2 , we establish some basic bounds on $v_{f}$. 
Lemma 7.3. Let $f \in C(\Omega), f \geq 0$. Then, in any set $\Omega_{\epsilon}:=\left\{x \in \Omega ; d_{\Omega}(x)>\epsilon\right\}$, $\epsilon>0, v_{f}$ satisfies the bounds

$$
0 \leq v_{f}(x) \leq\|f\|_{C\left(\Omega_{\epsilon}\right)} \tau(x) \prod_{i=1}^{n-1}\left(1+T \widetilde{K}_{-}\right) \quad \forall x \in \Omega_{\epsilon},
$$

where $T$ and $\widetilde{K}_{-}$are the constants defined by

$$
\begin{gathered}
T=\max \{\tau(x) ; x \in \partial \Omega\}, \\
\widetilde{K}_{-}=\max \left\{\left[\tilde{\kappa}_{i}(x)\right]_{-} ; x \in \partial \Omega, i=1, \ldots, n-1\right\},
\end{gathered}
$$

$[a]_{-}=\max \{0,-a\}$ being the negative part of a real number $a$.

Proof. By Lemma 5.4 and Corollary 6.8 we have that $1-\left(d_{\Omega}(x)+t\right) \tilde{\kappa}_{i}(x)>0$ for every $x \in \bar{\Omega} \backslash \bar{\Sigma}$ and $0 \leq t \leq \tau(x)$. Then the function $v_{f}$ is well defined and $v_{f} \geq 0$ in $\Omega$. In order to prove (17.8) it is enough to observe that

$$
\frac{1-\left(d_{\Omega}(x)+t\right) \tilde{\kappa}_{i}(x)}{1-d_{\Omega}(x) \tilde{\kappa}_{i}(x)} \leq 1+t\left[\tilde{\kappa}_{i}(x)\right]_{-}
$$

and that $x+t D \rho\left(D d_{\Omega}(x)\right) \in \Omega_{\epsilon}$ for every $x \in \Omega_{\epsilon}$ and $t \in[0, \tau(x)]$.

Proof of Theorem 7.2. The continuity of $v_{f}$ in $\Omega \backslash \bar{\Sigma}$ follows from the continuity of the functions $\tilde{\kappa}_{i}, i=1, \ldots, n$, in $\bar{\Omega} \backslash \bar{\Sigma}$, of the functions $f$ and $\tau$ in $\Omega$, and of the function $\Phi$ in $(\Omega \backslash \bar{\Sigma}) \times \mathbb{R}$. On the other hand, the continuity of $v_{f}$ on $\bar{\Sigma}$ is a consequence of (7.8).

Let $\varphi \in C_{c}^{\infty}(\Omega)$. From the change of variables formula (7.1) we have that

$$
\begin{aligned}
& \int_{\Omega} f(x) \varphi(x) d x \\
& =\int_{\partial \Omega} \rho(\nu(x))\left[\int_{0}^{l(x)} f(\Phi(x, t)) \varphi(\Phi(x, t)) \prod_{i=1}^{n-1}\left(1-t \tilde{\kappa}_{i}(x)\right) d t\right] d \mathcal{H}^{n-1}(x) .
\end{aligned}
$$

Let us compute the term in brackets, integrating by parts, and taking into account that $\varphi(\Phi(x, 0))=\varphi(x)=0$ for every $x \in \partial \Omega$. Setting $\Phi=\Phi(x, t)$, we get

$$
\begin{aligned}
& \int_{0}^{l(x)} f(\Phi) \varphi(\Phi) \prod_{i=1}^{n-1}\left(1-t \tilde{\kappa}_{i}(x)\right) d t \\
& =\int_{0}^{l(x)}\langle D \varphi(\Phi), D \rho(\nu(x))\rangle \int_{t}^{l(x)} f(\Phi(x, s)) \prod_{i=1}^{n-1}\left(1-s \tilde{\kappa}_{i}(x)\right) d s d t .
\end{aligned}
$$

Noticing that

$$
v_{f}(\Phi(x, t))=\int_{t}^{l(x)} f(\Phi(x, s)) \prod_{i=1}^{n-1} \frac{1-s \tilde{\kappa}_{i}(x)}{1-t \tilde{\kappa}_{i}(x)} d s \quad x \in \partial \Omega, t \in[0, l(x)),
$$

we obtain

$$
\begin{aligned}
& \int_{\Omega} f(x) \varphi(x) d x \\
& =\int_{\partial \Omega} \rho(\nu)\left[\int_{0}^{l(x)}\langle D \varphi(\Phi), D \rho(\nu)\rangle v_{f}(\Phi) \prod_{i=1}^{n-1}\left(1-t \tilde{\kappa}_{i}\right) d t\right] d \mathcal{H}^{n-1}(x) .
\end{aligned}
$$


Finally, again from (7.1),

$$
\int_{\Omega} f(x) \varphi(x) d x=\int_{\Omega} v_{f}(x)\left\langle D \rho\left(D d_{\Omega}(x)\right), D \varphi(x)\right\rangle d x,
$$

for every $\varphi \in C_{c}^{\infty}(\Omega)$.

\section{REFERENCES}

1. G. Alberti, On the structure of singular sets of convex functions, Calc. Var. Partial Differential Equations 2 (1994), 17-27. MR.1384392 (97e:26010)

2. B. Andrews, Volume-preserving anisotropic mean curvature flow, Indiana Univ. Math. J. 50 (2001), no. 2, 783-827. MR1871390 (2002m:53105)

3. D. Bao, S.-S. Chern, and Z. Shen, An introduction to Riemann-Finsler geometry, SpringerVerlag, New York, 2000.

4. M. Bardi and I. Capuzzo-Dolcetta, Optimal control and viscosity solutions of HamiltonJacobi-Bellman equations, Systems \& Control: Foundations \& Applications, Birkhäuser, Boston, 1997. MR1484411 (99e:49001)

5. G. Bouchitté and G. Buttazzo, Characterization of optimal shapes and masses through MongeKantorovich equation, J. Eur. Math. Soc. 3 (2001), 139-168. MR1831873 (2002c:49080)

6. P. Cannarsa, P. Cardaliaguet, G. Crasta, and E. Giorgieri, A boundary value problem for a PDE model in mass transfer theory: Representation of solutions and applications, Calc. Var. Partial Differential Equations 24 (2005), 431-457. MR2180861(2006i:35071)

7. P. Cannarsa, A. Mennucci, and C. Sinestrari, Regularity results for solutions of a class of Hamilton-Jacobi equations, Arch. Rational Mech. Anal. 140 (1997), 197-223. MR1486892 (99e:49032)

8. P. Cannarsa and C. Sinestrari, Semiconcave functions, Hamilton-Jacobi equations and optimal control, Progress in Nonlinear Differential Equations and their Applications, vol. 58, Birkhäuser, Boston, 2004. MR2041617 (2005e:49001)

9. P.T. Chruściel, J.H.G. Fu, G.J. Galloway, and R. Howard, On fine differentiability properties of horizons and applications to Riemannian geometry, J. Geom. Phys. 41 (2002), 1-12. MR.1872378 (2002k:53136)

10. F.H. Clarke, R.J. Stern, and P.R. Wolenski, Proximal smoothness and the lower-C $C^{2}$ property, J. Convex Anal. 2 (1995), 117-144. MR1363364 (96j:49014)

11. K. Deimling, Nonlinear functional analysis, Springer-Verlag, Berlin, 1985. MR0787404 (86j:47001)

12. L.C. Evans and R.F. Gariepy, Measure theory and fine properties of functions, CRC Press, Boca Raton, 1992. MR1158660 (93f:28001)

13. W.D. Evans and D.J. Harris, Sobolev embeddings for generalized ridged domains, Proc. London Math. Soc. 54 (1987), 141-175. MR0872254 (88b:46056)

14. J. Itoh and M. Tanaka, The Lipschitz continuity of the distance function to the cut locus, Trans. Amer. Math. Soc. 353 (2001), 21-40. MR1695025 (2001b:53029)

15. Y.Y. Li and L. Nirenberg, The distance function to the boundary, Finsler geometry and the singular set of viscosity solutions of some Hamilton-Jacobi equations, Commun. Pure Appl. Math. 58 (2005), 85-146. MR2094267 (2005k:35042)

16. P.L. Lions, Generalized solutions of Hamilton-Jacobi equations, Pitman, Boston, 1982.

17. C. Mantegazza and A.C. Mennucci, Hamilton-Jacobi equations and distance functions on Riemannian manifolds, Appl. Math. Optim. 47 (2003), 1-25. MR1941909 (2003h:49049)

18. A.C. Mennucci, Regularity and variationality of solutions to Hamilton-Jacobi equations. I. Regularity, ESAIM Control Optim. Calc. Var. 10 (2004), 426-451. MR2084331 (2005m:49050)

19. C. Pignotti, Rectifiability results for singular and conjugate points of optimal exit time problems, J. Math. Anal. Appl. 270 (2002), no. 2, 681-708. MR1916603 (2003h:49065)

20. G. Pisante, Sufficient conditions for the existence of viscosity solutions for nonconvex Hamiltonians, SIAM J. Math. Anal. 36 (2004), 186-203. MR2083857 (2005f:49078)

21. R.A. Poliquin, R.T. Rockafellar, and L. Thibault, Local differentiability of distance functions, Trans. Amer. Math. Soc. 352 (2000), 5231-5249. MR.1694378 (2001b:49024)

22. T. Sakai, Riemannian geometry, Translations of Mathematical Monographs, vol. 149, American Mathematical Society, Providence, RI, 1996. MR1390760 (97f:53001) 
23. R. Schneider, Convex bodies: the Brunn-Minkowski theory, Cambridge Univ. Press, Cambridge, 1993. MR1216521 (94d:52007)

24. Z. Shen, Geometric meanings of curvatures in Finsler geometry, Rend. Circ. Mat. Palermo (2) Suppl. 66 (2001), 165-178, Proceedings of the 20th Winter School "Geometry and Physics" (Srní, 2000). MR1826690 (2002e:53029)

25. J.A. Thorpe, Elementary topics in differential geometry, Springer-Verlag, New York, 1979. MR0528129 (80e:53001)

Dipartimento di Matematica "G. Castelnuovo", Univ. di Roma I, P.le A. Moro 2 00185 Roma, ITALY

E-mail address: crasta@mat.uniroma1.it

Dipartimento di Matematica "G. Castelnuovo", Univ. di Roma i, P.le A. Moro 2 00185 Roma, ITALY

E-mail address: malusa@mat.uniroma1.it 